\title{
Hybrid Algorithms of Common Solutions of Generalized Mixed Equilibrium Problems and the Common Variational Inequality Problems with Applications
}

\section{Thanyarat Jitpeera, Uamporn Witthayarat, and Poom Kumam}

Department of Mathematics, Faculty of Science, King Mongkut's University of Technology Thonburi (KMUTT), Bangmod, Bangkok 10140, Thailand

Correspondence should be addressed to Poom Kumam, poom.kum@kmutt.ac.th

Received 5 January 2011; Accepted 20 February 2011

Academic Editor: Tomonari Suzuki

Copyright (C) 2011 Thanyarat Jitpeera et al. This is an open access article distributed under the Creative Commons Attribution License, which permits unrestricted use, distribution, and reproduction in any medium, provided the original work is properly cited.

\begin{abstract}
We introduce new iterative algorithms by hybrid method for finding a common element of the set of solutions of fixed points of infinite family of nonexpansive mappings, the set of common solutions of generalized mixed equilibrium problems, and the set of common solutions of the variational inequality with inverse-strongly monotone mappings in a real Hilbert space. We prove the strong convergence of the proposed iterative method under some suitable conditions. Finally, we apply our results to complementarity problems and optimization problems. Our results improve and extend the results announced by many others.
\end{abstract}

\section{Introduction}

Throughout this paper, let $H$ be a real Hilbert space with inner product $\langle\cdot, \cdot\rangle$ and norm $\|\cdot\|$, and let $C$ be a nonempty closed convex subset of $H$. A mapping $T: C \rightarrow C$ is called nonexpansive if $\|T x-T y\| \leq\|x-y\|$, for all $x, y \in C$. The set of fixed points of $T$ denoted by $F(T)$; that is, $F(T)=\{x \in C: T x=x\}$. If $C \subset H$ is bounded, closed, and convex and $T$ is a nonexpansive mapping of $C$ into itself, then $F(T) \neq \emptyset$; see, for instance, [1]. Let $F$ be a bifunction of $C \times C$ into $\mathbb{R}$, where $\mathbb{R}$ is the set of real numbers, $A: C \rightarrow H$ a mapping, and $\varphi: C \rightarrow \mathbb{R}$ a real-valued function. The generalized mixed equilibrium problem is for finding $x \in C$ such that

$$
F(x, y)+\langle A x, y-x\rangle+\varphi(y)-\varphi(x) \geq 0, \quad \forall y \in C
$$


The set of solutions of (1.1) is denoted by $\operatorname{GMEP}(F, \varphi, A)$; that is,

$$
\operatorname{GMEP}(F, \varphi, A)=\{x \in C: F(x, y)+\langle A x, y-x\rangle+\varphi(y)-\varphi(x) \geq 0, \forall y \in C\}
$$

The generalized mixed equilibrium problem include fixed point problems, optimization problems, variational inequalities problems, Nash equilibrium problems, noncooperative games, economics, and the equilibrium problems as special cases [2-7].

In particular, if $A \equiv 0$, the problem (1.1) is reduced into the mixed equilibrium problem [8] for finding $x \in C$ such that

$$
F(x, y)+\varphi(y)-\varphi(x) \geq 0, \quad \forall y \in C
$$

The set of solutions of (1.3) is denoted by $\operatorname{MEP}(F, \varphi)$. such that

If $\varphi \equiv 0,(1.1)$ is reduced into the generalized equilibrium problem [9] for finding $x \in C$

$$
F(x, y)+\langle A x, y-x\rangle \geq 0, \quad \forall y \in C
$$

The set of solutions of (1.4) is denoted by $\operatorname{GEP}(F, A)$, which this problem was studied by $\mathrm{S}$. Takahashi and W. Takahashi [10].

If $A \equiv 0$ and $\varphi \equiv 0$, then the generalized mixed equilibrium problem (1.1) becomes the following equilibrium problem which is to find $x \in C$ such that

$$
F(x, y) \geq 0, \quad \forall y \in C
$$

The set of solutions of (1.5) is denoted by $\mathrm{EP}(F)$. Many problems in applied sciences, such as numerous problems in physics, optimization, and economics reduce into finding a solution of (1.5). Some methods have been proposed to solve the generalized mixed equilibrium problems, equilibrium problems, and fixed point problems $([2,6,11-29])$ and references therein. If $F \equiv 0$ and $\varphi \equiv 0$, then the generalized mixed equilibrium problem (1.1) becomes the following variational inequality problem, denoted by $\operatorname{VI}(C, A)$, is to find $x \in C$ such that

$$
\langle A x, y-x\rangle \geq 0, \quad \forall y \in C
$$

The variational inequality problem has been extensively studied in the literature. See, for example $[30,31]$ and the references therein. A mapping $A$ of $C$ into $H$ is called monotone if

$$
\langle A x-A y, x-y\rangle \geq 0, \quad \forall x, y \in C
$$

$A$ is called an $\alpha$-inverse-strongly monotone if there exists a positive real number $\alpha>0$ such that

$$
\langle A x-A y, x-y\rangle \geq \alpha\|A x-A y\|^{2}, \quad \forall x, y \in C .
$$


In 2008, Takahashi et al. [32] introduced an iterative method for finding the set of fixed point by Hybrid method in Hilbert spaces. Starting with $C_{1}=C, x_{1}=P_{C_{1}} x_{0}$, define sequence $\left\{x_{n}\right\},\left\{y_{n}\right\}$ as follows:

$$
\begin{gathered}
y_{n}=\alpha_{n} x_{n}+\left(1-\alpha_{n}\right) T x_{n}, \quad n \geq 1, \\
C_{n+1}=\left\{z \in C_{n}:\left\|y_{n}-z\right\| \leq\left\|x_{n}-z\right\|\right\}, \quad n \geq 1, \\
x_{n+1}=P_{C_{n+1}} x_{0}, \quad n \geq 1,
\end{gathered}
$$

where $P_{C}$ is a metric projection of $H$ onto $C$ and $T$ is a nonexpansive mapping of $C$ into itself. They proved that if the sequence $\left\{\alpha_{n}\right\}$ of parameters satisfies appropriate conditions, then $\left\{x_{n}\right\}$ generated by (1.9) converges strongly to $P_{F(T)} x_{0}$. In 2009, Kumam [20] introduced an iterative method for finding a common element of the set of common fixed points of nonexpansive mapping, the set of solutions of a variational inequality problem, and the set of solutions of an equilibrium problem in Hilbert spaces. Starting with an arbitrary $C_{1}=C$, $x_{1}=P_{C_{1}} x_{0}$, define sequence $\left\{x_{n}\right\},\left\{z_{n}\right\}$ as follows:

$$
\begin{gathered}
F\left(z_{n}, y\right)+\frac{1}{r_{n}}\left\langle y-z_{n}, z_{n}-x_{n}\right\rangle \geq 0, \quad \forall y \in C, \\
y_{n}=\alpha_{n} x_{n}+\left(1-\alpha_{n}\right) T P_{C}\left(z_{n}-\lambda_{n} B z_{n}\right), \quad n \geq 1, \\
C_{n+1}=\left\{z \in C_{n}:\left\|y_{n}-z\right\| \leq\left\|x_{n}-z\right\|\right\}, \quad n \geq 1, \\
x_{n+1}=P_{C_{n+1}} x_{0}, \quad n \geq 1,
\end{gathered}
$$

where $T$ is a nonexpansive mapping of $C$ into itself and $B$ is a $\beta$-inverse-strongly monotone mapping of $C$ into $H$. He proved that if the sequences $\left\{\alpha_{n}\right\},\left\{r_{n}\right\}$, and $\left\{\lambda_{n}\right\}$ of parameters satisfies appropriate conditions, then $\left\{x_{n}\right\}$ generated by (1.10) converges strongly to $P_{F(T) \cap E P(F) \cap V I(C, B)} x_{0}$. In 2010, Kangtunyakarn [33] introduced a new method for a common of generalized equilibrium problems, common of variational inequality problems, and fixed point problems by using $S$-mapping generated by a finite family of nonexpansive mappings and real numbers in Hilbert spaces. Starting with an arbitrary $x_{1}, u, v$ in $C$, define the sequences $\left\{x_{n}\right\},\left\{y_{n}\right\}$ as follows:

$$
\begin{gathered}
F\left(u_{n}, u\right)+\left\langle A x_{n}, u-u_{n}\right\rangle+\frac{1}{r_{n}}\left\langle u-u_{n}, u_{n}-x_{n}\right\rangle \geq 0, \quad \forall u \in C, \\
G\left(v_{n}, v\right)+\left\langle B x_{n}, v-v_{n}\right\rangle+\frac{1}{s_{n}}\left\langle v-v_{n}, v_{n}-x_{n}\right\rangle \geq 0, \quad \forall v \in C, \\
y_{n}=\delta_{n} P_{C}\left(u_{n}-\lambda_{n} A u_{n}\right)+\left(1-\delta_{n}\right) P_{C}\left(v_{n}-\eta_{n} B v_{n}\right), \quad n \geq 1, \\
x_{n+1}=\alpha_{n} f\left(x_{n}\right)+\beta_{n} x_{n}+\gamma_{n} S_{n} y_{n}, \quad \forall n \geq 1,
\end{gathered}
$$

where $S_{n}$ is the $S$-mapping and $A, B$ are $\alpha, \beta$-inverse-strongly monotone mappings of $C$ into $H$, respectively. He proved that if the sequences $\left\{\alpha_{n}\right\},\left\{\beta_{n}\right\},\left\{\gamma_{n}\right\},\left\{r_{n}\right\},\left\{s_{n}\right\},\left\{\eta_{n}\right\}$, and $\left\{\lambda_{n}\right\}$ of parameters satisfies appropriate conditions, then $\left\{x_{n}\right\}$ generated by (1.11) converges strongly to $P_{\Im}:=\cap_{n=1}^{\infty} F\left(S_{n}\right) \cap \operatorname{MEP}(F, A) \cap \mathrm{MEP}(G, B) \cap \mathrm{VI}(C, A) \cap V \mathrm{I}(C, B) x_{1}$. 
Recently, Shehu [34] motivated Chantarangsi et al. [35] who studied the problem of approximating a common element of the set of fixed points of an infinite family of nonexpansive mapping, the set of common solutions of generalized mixed equilibrium problems, and the set of solutions to a variational inequality problem in a real Hilbert spaces.

In this paper, motivated by the above results, we present a new hybrid iterative scheme for finding a common element of the set of solutions of a common of generalized mixed equilibrium problems, the common solutions of the variational inequality for inversestrongly monotone mapping, and the set of fixed points of infinite family of nonexpansive mappings in the set of Hilbert spaces. Then, we prove strong convergence theorems under some mild conditions. Finally, we give some applications of our results. The results presented in this paper generalize, extend, and improve the results of Takahashi et al. [32], Kumam [20], Kangtunyakarn [33], and many authors.

\section{Preliminaries}

Let $H$ be a real Hilbert space with norm $\|\cdot\|$ and inner product $\langle\cdot, \cdot\rangle$, and let $C$ be a closed convex subset of $H$. When $\left\{x_{n}\right\}$ is a sequence in $H, x_{n} \rightarrow x$ means $\left\{x_{n}\right\}$ converges weakly to $x$, and $x_{n} \rightarrow x$ means $\left\{x_{n}\right\}$ converges strongly to $x$. In a real Hilbert space $H$, we have

$$
\begin{gathered}
\|x-y\|^{2}=\|x\|^{2}-\|y\|^{2}-2\langle x-y, y\rangle \\
\|\lambda x+(1-\lambda) y\|^{2}=\lambda\|x\|^{2}+(1-\lambda)\|y\|^{2}-\lambda(1-\lambda)\|x-y\|^{2}
\end{gathered}
$$

for all $x, y \in H$ and $\lambda \in[0,1]$. For every point $x \in H$, there exists a unique nearest point in $C$, denoted by $P_{C} x$, such that

$$
\left\|x-P_{C} x\right\| \leq\|x-y\|, \quad \forall y \in C
$$

$P_{C}$ is called the metric projection of $H$ onto $C$. It is well known that $P_{C}$ is a nonexpansive mapping of $H$ onto $C$ and satisfies

$$
\left\langle x-y, P_{C} x-P_{C} y\right\rangle \geq\left\|P_{C} x-P_{C} y\right\|^{2}, \quad \forall x, y \in H
$$

Moreover, $P_{C} x$ is characterized by the following properties: $P_{C} x \in C$,

$$
\begin{gathered}
\left\langle x-P_{C} x, y-P_{C} x\right\rangle \leq 0, \\
\|x-y\|^{2} \geq\left\|x-P_{C} x\right\|^{2}+\left\|y-P_{C} x\right\|^{2},
\end{gathered}
$$

for all $x \in H, y \in C$. It is also known that $H$ satisfies the Opial condition; for any sequence $\left\{x_{n}\right\}$ with $x_{n} \rightarrow x$, the inequality

$$
\liminf _{n \rightarrow \infty}\left\|x_{n}-x\right\|<\liminf _{n \rightarrow \infty}\left\|x_{n}-y\right\|
$$

holds for every $y \in H$ with $y \neq x$. 
It is obvious that any $\alpha$-inverse-strongly monotone mapping $A$ is $(1 / \alpha)$-Lipschitz monotone and continuous mapping. We also have that for all $x, y \in H$ and $\lambda>0$,

$$
\begin{aligned}
\|(I-r A) x-(I-r A) y\|^{2} & =\|(x-y)-r(A x-A y)\|^{2} \\
& =\|x-y\|^{2}-2 r\langle x-y, A x-A y\rangle+r^{2}\|A x-A y\|^{2} \\
& \leq\|x-y\|^{2}+r(r-2 \alpha)\|A x-A y\|^{2} .
\end{aligned}
$$

So, if $r \leq 2 \alpha$, then $I-r A$ is a nonexpansive mapping of $C$ into $H$.

For solving the generalized mixed equilibrium problem, let us assume that the bifunction $F: C \times C \rightarrow \mathbb{R}$, the nonlinear mapping $A: C \rightarrow H$ is continuous monotone, $\varphi: C \rightarrow \mathbb{R}$ is convex, and lower semicontinuous satisfies the following conditions:

(A1) $F(x, x)=0$ for all $x \in C$,

(A2) $F$ is monotone; that is, $F(x, y)+F(y, x) \leq 0$ for any $x, y \in C$,

(A3) $F$ is upper-hemicontinuous; that is, for each $u, x, y \in C$,

$$
\limsup _{t \rightarrow 0^{+}} F(t u+(1-t) x, y) \leq F(x, y)
$$

(A4) $F(x, \cdot)$ is convex and lower semicontinuous for each $x \in C$,

(B1) For each $x \in H$ and $r>0$, there exists a bounded subset $D_{x} \subseteq C$ and $y_{x} \in C \cap$ $\operatorname{dom}(\varphi)$ such that for any $u \in C \backslash D_{x}$,

$$
F\left(u, y_{x}\right)+\left\langle A u, y_{x}-u\right\rangle+\varphi\left(y_{x}\right)+\frac{1}{r}\left\langle y_{x}-u, u-x\right\rangle\langle\varphi(u)
$$

(B2) $C$ is a bounded set.

The following lemma appears implicitly in [2]. We need the following lemmas for proving our main result.

Lemma 2.1 (see [2]). Let $C$ be a nonempty closed convex subset of $H$, and let $F$ be a bifunction of $C \times C$ into $\mathbb{R}$ satisfying (A1)-(A4). Let $r>0$ and $x \in H$. Then, there exists $u \in C$ such that

$$
F(u, y)+\frac{1}{r}\langle y-u, u-x\rangle \geq 0, \quad \forall y \in C
$$

The following lemma was also given in [36].

Lemma 2.2 (see [36]). Assume that $F: C \times C \rightarrow \mathbb{R}$ satisfies (A1)-(A4). For $r>0$ and $x \in H$, define a mapping $K_{r}: H \rightarrow C$ as follows:

$$
K_{r}(x)=\left\{u \in C: F(u, y)+\frac{1}{r}\langle y-u, u-x\rangle \geq 0, \forall y \in C\right\}
$$


for all $x \in H$. Then, the following hold:

(1) $K_{r}$ is single-valued,

(2) $K_{r}$ is firmly nonexpansive, that is, for any $x, y \in H,\left\|K_{r} x-K_{r} y\right\|^{2} \leq\left\langle K_{r} x-K_{r} y, x-y\right\rangle$,

(3) $F\left(K_{r}\right)=\mathrm{EP}(F)$,

(4) $\mathrm{EP}(F)$ is closed and convex.

Lemma 2.3 (see [37]). Let $C$ be a nonempty closed convex subset of a real Hilbert space $H$. Let $F: C \times C \rightarrow \mathbb{R}$ be a bifunction mapping satisfies (A1)-(A4), and let $\varphi: C \rightarrow \mathbb{R}$ be convex and lower semicontinuous such that $C \cap \operatorname{dom} \varphi \neq \emptyset$. Assume that either (B1) or (B2) holds. For $r>0$ and $x \in H$, there exists $u \in C$ such that

$$
F(u, y)+\varphi(y)-\varphi(u)+\frac{1}{r}\langle y-u, u-x\rangle
$$

Define a mapping $K_{r}: H \rightarrow C$ as follows:

$$
T_{r}^{(F, \varphi)}(x)=\left\{u \in C: F(u, y)+\varphi(y)-\varphi(u)+\frac{1}{r}\langle y-u, u-x\rangle \geq 0, \forall y \in C\right\}
$$

for all $x \in H$. Then, the following hold:

(1) $T_{r}^{(F, \varphi)}$ is single-valued,

(2) $T_{r}^{(F, \varphi)}$ is firmly nonexpansive, that is, for any $x, y \in H,\left\|T_{r}^{(F, \varphi)} x-T_{r}^{(F, \varphi)} y\right\|^{2} \leq\left\langle T_{r}^{(F, \varphi)} x-\right.$ $\left.T_{r}^{(F, \varphi)} y, x-y\right\rangle$,

(3) $F\left(T_{r}^{(F, \varphi)}\right)=\operatorname{MEP}(F, \varphi)$,

(4) $\operatorname{MEP}(F, \varphi)$ is closed and convex.

Lemma 2.4 (see [38]). Assume that $\left\{a_{n}\right\}$ is a sequence of nonnegative real numbers such that

$$
a_{n+1} \leq\left(1-\alpha_{n}\right) a_{n}+\delta_{n}, \quad n \geq 0,
$$

where $\left\{\alpha_{n}\right\}$ is a sequence in $(0,1)$ and $\left\{\delta_{n}\right\}$ is a sequence in $\mathbb{R}$ such that

(1) $\sum_{n=1}^{\infty} \alpha_{n}=\infty$,

(2) $\lim \sup _{n \rightarrow \infty}\left(\delta_{n} / \alpha_{n}\right) \leq 0$ or $\sum_{n=1}^{\infty}\left|\delta_{n}\right|<\infty$.

Then, $\lim _{n \rightarrow \infty} a_{n}=0$.

\section{Main Result}

In this section, we prove a strong convergence theorem for finding a common element of the set of solutions of a common of generalized mixed equilibrium problems, the common solutions of the variational inequality for inverse-strongly monotone mapping, and 
the set of fixed points of infinite family of nonexpansive mappings in the set of Hilbert spaces.

Theorem 3.1. Let $C$ be a nonempty closed convex subset of a real Hilbert space $H$. Let $F_{1}, F_{2}$ be a bifunction of $C \times C$ into real numbers $\mathbb{R}$ satisfying (A1)-(A4), and let $\varphi_{1}, \varphi_{2}: C \rightarrow \mathbb{R} \cup\{+\infty\}$ be a proper lower semicontinuous and convex function. Let $A, B, D$, and $E$ be $\alpha, \beta, \delta$, and $\eta$ inverse-strongly monotone mapping of $C$ into $H$, respectively. Let $\left\{T_{i}\right\}_{i=1}^{\infty}$ be an infinite nonexpansive mapping such that $\Theta:=\bigcap_{i=1}^{\infty} F\left(T_{i}\right) \cap \operatorname{GMEP}\left(F_{1}, \varphi_{1}, A\right) \cap \operatorname{GMEP}\left(F_{2}, \varphi_{2}, B\right) \cap \operatorname{VI}(C, D) \cap \operatorname{VI}(C, E) \neq \emptyset$. Assume that either (B1) or (B2) holds. Let $\left\{x_{n}\right\}$ be a sequence generated by $x_{0} \in C, C_{1, i}=C$, $C_{1}=\cap_{i=1}^{\infty} C_{1, i}, x_{1}=P_{C_{1}} x_{0}$ and

$$
\begin{gathered}
t_{n}=T_{r_{n}}^{\left(F_{1}, \varphi_{1}\right)}\left(x_{n}-r_{n} A x_{n}\right), \\
u_{n}=T_{s_{n}}^{\left(F_{2}, \varphi_{2}\right)}\left(x_{n}-s_{n} B x_{n}\right), \\
w_{n}=\xi_{n} P_{C}\left(u_{n}-\lambda_{n} D u_{n}\right)+\left(1-\xi_{n}\right) P_{C}\left(t_{n}-\mu_{n} E t_{n}\right), \\
y_{n, i}=\alpha_{n, i} x_{0}+\left(1-\alpha_{n, i}\right) T_{i} w_{n}, \\
C_{n+1, i}=\left\{z \in C_{n, i}:\left\|y_{n, i}-z\right\|^{2} \leq\left\|x_{n}-z\right\|^{2}+\alpha_{n, i}\left(\left\|x_{0}\right\|^{2}+2\left\langle w_{n}-x_{0}, z\right\rangle\right)\right\}, \\
C_{n+1}=\bigcap_{i=1}^{\infty} C_{n+1, i}, \\
x_{n+1}=P_{C_{n+1}} x_{0},
\end{gathered}
$$

for every $n \geq 0$, where $\left\{r_{n}\right\},\left\{s_{n}\right\} \subset(0, \infty), \lambda_{n} \in(0,2 \delta)$ and $\mu_{n} \in(0,2 \eta)$ satisfying the following conditions:

(i) $0<a \leq r_{n} \leq b<2 \alpha$,

(ii) $0<c \leq s_{n} \leq d<2 \beta$,

(iii) $\lim _{n \rightarrow \infty} \alpha_{n, i}=0$,

(iv) $\lim _{n \rightarrow \infty} \xi_{n}=\xi \in(0,1)$,

(v) $0<e \leq \lambda_{n} \leq f<2 \delta$,

(vi) $0<g \leq \mu_{n} \leq j<2 \eta$.

Then, $\left\{x_{n}\right\}$ converges strongly to $P_{\Theta} x_{0}$. 
Proof. Let $p \in \Theta$, then $p=T_{r_{n}}^{\left(F_{1}, \varphi_{1}\right)}\left(p-r_{n} A p\right), p=T_{s_{n}}^{\left(F_{2}, \varphi_{2}\right)}\left(p-s_{n} B p\right), p=P_{C}\left(p-\lambda_{n} D p\right)$, and $p=P_{C}\left(p-\mu_{n} E p\right)$. By nonexpansiveness of $P_{C}, T_{r_{n}}^{\left(F_{1}, \varphi_{1}\right)}$, and $T_{s_{n}}^{\left(F_{2}, \varphi_{2}\right)}$, we have

$$
\begin{aligned}
& \left\|w_{n}-p\right\|^{2} \\
& =\left\|\xi_{n} P_{C}\left(u_{n}-\lambda_{n} D u_{n}\right)+\left(1-\xi_{n}\right) P_{C}\left(t_{n}-\mu_{n} E t_{n}\right)-\xi_{n} P_{C}\left(p-\lambda_{n} D p\right)-\left(1-\xi_{n}\right) P_{C}\left(p-\mu_{n} E p\right)\right\|^{2} \\
& =\left\|\xi_{n}\left\{P_{C}\left(u_{n}-\lambda_{n} D u_{n}\right)-P_{C}\left(p-\lambda_{n} D p\right)\right\}+\left(1-\xi_{n}\right)\left\{P_{C}\left(t_{n}-\mu_{n} E t_{n}\right)-P_{C}\left(p-\mu_{n} E p\right)\right\}\right\|^{2} \\
& \leq \xi_{n}\left\|\left(u_{n}-\lambda_{n} D u_{n}\right)-\left(p-\lambda_{n} D p\right)\right\|^{2}+\left(1-\xi_{n}\right)\left\|\left(t_{n}-\mu_{n} E t_{n}\right)-\left(p-\mu_{n} E p\right)\right\|^{2} \\
& =\xi_{n}\left\|\left(u_{n}-p\right)-\lambda_{n}\left(D u_{n}-D p\right)\right\|^{2}+\left(1-\xi_{n}\right)\left\|\left(t_{n}-p\right)-\mu_{n}\left(E t_{n}-E p\right)\right\|^{2} \\
& =\xi_{n}\left\{\left\|u_{n}-p\right\|^{2}-\lambda_{n}\left(2 \delta-\lambda_{n}\right)\left\|D u_{n}-D p\right\|^{2}\right\} \\
& +\left(1-\xi_{n}\right)\left\{\left\|t_{n}-p\right\|^{2}-\mu_{n}\left(2 \eta-\mu_{n}\right)\left\|E t_{n}-E p\right\|^{2}\right\} \\
& \leq \xi_{n}\left\{\left\|T_{s_{n}}^{\left(F_{2}, \varphi_{2}\right)}\left(x_{n}-s_{n} B x_{n}\right)-T_{s_{n}}^{\left(F_{2}, \varphi_{2}\right)}\left(p-s_{n} B p\right)\right\|^{2}-\lambda_{n}\left(2 \delta-\lambda_{n}\right)\left\|D u_{n}-D p\right\|^{2}\right\} \\
& +\left(1-\xi_{n}\right)\left\{\left\|T_{r_{n}}^{\left(F_{1}, \varphi_{1}\right)}\left(x_{n}-r_{n} A x_{n}\right)-T_{r_{n}}^{\left(F_{1}, \varphi_{1}\right)}\left(p-r_{n} A p\right)\right\|^{2}-\mu_{n}\left(2 \eta-\mu_{n}\right)\left\|E t_{n}-E p\right\|^{2}\right\} \\
& \leq \xi_{n}\left\{\left\|\left(x_{n}-s_{n} B x_{n}\right)-\left(p-s_{n} B p\right)\right\|^{2}\right\}+\left(1-\xi_{n}\right)\left\{\left\|\left(x_{n}-r_{n} A x_{n}\right)-\left(p-r_{n} A p\right)\right\|^{2}\right\} \\
& \leq \xi_{n}\left\|x_{n}-p\right\|^{2}+\left(1-\xi_{n}\right)\left\|x_{n}-p\right\|^{2} \\
& \leq\left\|x_{n}-p\right\|^{2} \text {. }
\end{aligned}
$$

Since both $I-r_{n} A$ and $I-s_{n} B$ are nonexpansive for each $n \geq 1$ and (2.7), we have

$$
\begin{aligned}
\left\|u_{n}-p\right\|^{2} & =\left\|T_{s_{n}}^{\left(F_{2}, \varphi_{2}\right)}\left(I-s_{n} B\right) x_{n}-T_{s_{n}}^{\left(F_{2}, \varphi_{2}\right)}\left(I-s_{n} B\right) p\right\|^{2} \\
& \leq\left\|\left(I-s_{n} B\right) x_{n}-\left(I-s_{n} B\right) p\right\|^{2} \\
& \leq\left\|x_{n}-p\right\|^{2}+s_{n}\left(s_{n}-2 \beta\right)\left\|B x_{n}-B p\right\|^{2} \\
& \leq\left\|x_{n}-p\right\|^{2}, \\
\left\|t_{n}-p\right\|^{2} & =\left\|T_{r_{n}}^{\left(F_{1}, \varphi_{1}\right)}\left(I-r_{n} A\right) x_{n}-T_{r_{n}}^{\left(F_{1}, \varphi_{1}\right)}\left(I-r_{n} A\right) p\right\|^{2} \\
& \leq\left\|\left(I-r_{n} A\right) x_{n}-\left(I-r_{n} A\right) p\right\|^{2} \\
& \leq\left\|x_{n}-p\right\|^{2}+r_{n}\left(r_{n}-2 \alpha\right)\left\|A x_{n}-A p\right\|^{2} \\
& \leq\left\|x_{n}-p\right\|^{2} .
\end{aligned}
$$

Therefore, we obtain $\left\|u_{n}-p\right\| \leq\left\|x_{n}-p\right\|$ and $\left\|t_{n}-p\right\| \leq\left\|x_{n}-p\right\|$. 
Next, we will divide the proof into four steps.

Step 1. We show that $\left\{x_{n}\right\}$ is well defined. Let $n=1$, then $C_{1, i}=C$ is closed and convex for each $i \geq 1$. Suppose that $C_{n, i}$ is closed convex for some $n>1$. Then, by definition of $C_{n+1, i}$, we know that $C_{n+1, i}$ is closed convex for $n \geq 1$. Hence, $C_{n, i}$ is closed convex for $n \geq 1$ and for each $i \geq 1$. This implies that $C_{n}$ is closed convex for $n \geq 1$. Moreover, we show that $\Theta \subset C_{n}$. For $n=1, \Theta \subset C=C_{1, i}$. For $n \geq 2$, let $p \in \Theta$. Then,

$$
\begin{aligned}
\left\|y_{n, i}-p\right\|^{2} & =\left\|\alpha_{n, i}\left(x_{0}-p\right)^{2}+\left(1-\alpha_{n, i}\right)\left(T_{i} w_{n}-p\right)\right\|^{2} \\
& \leq \alpha_{n, i}\left\|x_{0}-p\right\|^{2}+\left(1-\alpha_{n, i}\right)\left\|w_{n}-p\right\|^{2} \\
& =\left\|w_{n}-p\right\|^{2}+\alpha_{n, i}\left(\left\|x_{0}-p\right\|^{2}-\left\|w_{n}-p\right\|^{2}\right) \\
& \leq\left\|x_{n}-p\right\|^{2}+\alpha_{n, i}\left(\left\|x_{0}\right\|^{2}+2\left\langle w_{n}-x_{0}, p\right\rangle\right)
\end{aligned}
$$

which shows that $p \in C_{n, i}$, for all $n \geq 2$, for all $i \geq 1$. So, $\Theta \subset C_{n, i}$, for all $n \geq 1$, for all $i \geq 1$. Therefore, it follows that $\emptyset \neq \Theta \subset C_{n}$, for all $n \geq 1$. This implies that $\left\{x_{n}\right\}$ is well defined.

Step 2. We claim that $\lim _{n \rightarrow \infty}\left\|x_{n+1}-x_{n}\right\|=0$ and $\lim _{n \rightarrow \infty}\left\|y_{n, i}-x_{n}\right\|=0$.

From $x_{n}=P_{C_{n}} x_{0}$, we get

$$
\left\langle x_{0}-x_{n}, x_{n}-y\right\rangle \geq 0,
$$

for each $y \in C_{n}$. Since $\Theta \subset C_{n}$, we have

$$
\left\langle x_{0}-x_{n}, x_{n}-p\right\rangle \geq 0 \text { for each } p \in \Theta, n \in \mathbb{N}
$$

Hence, for $p \in \Theta$, we obtain

$$
\begin{aligned}
0 & \leq\left\langle x_{0}-x_{n}, x_{n}-p\right\rangle \\
& =\left\langle x_{0}-x_{n}, x_{n}-x_{0}+x_{0}-p\right\rangle \\
& =-\left\langle x_{0}-x_{n}, x_{0}-x_{n}\right\rangle+\left\langle x_{0}-x_{n}, x_{0}-p\right\rangle \\
& \leq-\left\|x_{0}-x_{n}\right\|^{2}+\left\|x_{0}-x_{n}\right\|\left\|x_{0}-p\right\| .
\end{aligned}
$$

It follows that

$$
\left\|x_{0}-x_{n}\right\| \leq\left\|x_{0}-p\right\|, \quad \forall p \in \Theta, n \in \mathbb{N} .
$$

From $x_{n}=P_{C_{n}} x_{0}$ and $x_{n+1}=P_{C_{n+1}} x_{0} \in C_{n+1} \subset C_{n}$, we have

$$
\left\langle x_{0}-x_{n}, x_{n}-x_{n+1}\right\rangle \geq 0
$$


For $n \in \mathbb{N}$, we compute

$$
\begin{aligned}
0 & \leq\left\langle x_{0}-x_{n}, x_{n}-x_{n+1}\right\rangle \\
& =\left\langle x_{0}-x_{n}, x_{n}-x_{0}+x_{0}-x_{n+1}\right\rangle \\
& =-\left\langle x_{0}-x_{n}, x_{0}-x_{n}\right\rangle+\left\langle x_{0}-x_{n}, x_{0}-x_{n+1}\right\rangle \\
& \leq-\left\|x_{0}-x_{n}\right\|^{2}+\left\langle x_{0}-x_{n}, x_{0}-x_{n+1}\right\rangle \\
& \leq-\left\|x_{0}-x_{n}\right\|^{2}+\left\|x_{0}-x_{n}\right\|\left\|x_{0}-x_{n+1}\right\|
\end{aligned}
$$

and then

$$
\left\|x_{0}-x_{n}\right\| \leq\left\|x_{0}-x_{n+1}\right\|, \quad \forall n \in \mathbb{N} .
$$

Thus, the sequence $\left\{\left\|x_{n}-x_{0}\right\|\right\}$ is a bounded and nondecreasing sequence, so $\lim _{n \rightarrow \infty}\left\|x_{n}-x_{0}\right\|$ exists. That is, there exists $m$ such that

$$
m=\lim _{n \rightarrow \infty}\left\|x_{n}-x_{0}\right\|
$$

Hence, $\left\{x_{n}\right\}$ is bounded and so are $\left\{A x_{n}\right\},\left\{B x_{n}\right\},\left\{u_{n}\right\},\left\{D u_{n}\right\},\left\{t_{n}\right\},\left\{E t_{n}\right\},\left\{w_{n}\right\},\left\{T_{i} w_{n}\right\}$, and $\left\{y_{n, i}\right\}$ for $i=1,2, \ldots$, and $n \geq 1$. From (3.9), we get

$$
\begin{aligned}
\left\|x_{n}-x_{n+1}\right\|^{2} & =\left\|x_{n}-x_{0}+x_{0}-x_{n+1}\right\|^{2} \\
& =\left\|x_{n}-x_{0}\right\|^{2}+2\left\langle x_{n}-x_{0}, x_{0}-x_{n+1}\right\rangle+\left\|x_{0}-x_{n+1}\right\|^{2} \\
& =\left\|x_{n}-x_{0}\right\|^{2}+2\left\langle x_{n}-x_{0}, x_{0}-x_{n}+x_{n}-x_{n+1}\right\rangle+\left\|x_{0}-x_{n+1}\right\|^{2} \\
& =\left\|x_{n}-x_{0}\right\|^{2}-2\left\langle x_{n}-x_{0}, x_{n}-x_{0}\right\rangle+2\left\langle x_{n}-x_{0}, x_{n}-x_{n+1}\right\rangle+\left\|x_{0}-x_{n+1}\right\|^{2} \\
& =-\left\|x_{n}-x_{0}\right\|^{2}+2\left\langle x_{n}-x_{0}, x_{n}-x_{n+1}\right\rangle+\left\|x_{0}-x_{n+1}\right\|^{2} \\
& \leq-\left\|x_{n}-x_{0}\right\|^{2}+\left\|x_{0}-x_{n+1}\right\|^{2} .
\end{aligned}
$$

By (3.12), we obtain

$$
\lim _{n \rightarrow \infty}\left\|x_{n}-x_{n+1}\right\|=0
$$

Since $x_{n+1}=P_{C_{n+1}} x_{0} \in C_{n+1} \subset C_{n}$, we have

$$
\left\|y_{n, i}-x_{n+1}\right\|^{2} \leq\left\|x_{n}-x_{n+1}\right\|^{2}+\alpha_{n, i}\left(\left\|x_{0}\right\|^{2}+2\left\langle w_{n}-x_{0}, x_{n+1}\right\rangle\right) .
$$

By (iii) and (3.14), we get

$$
\lim _{n \rightarrow \infty}\left\|y_{n, i}-x_{n+1}\right\|=0 .
$$


Fixed Point Theory and Applications

It follows that

$$
\left\|y_{n, i}-x_{n}\right\| \leq\left\|y_{n, i}-x_{n+1}\right\|+\left\|x_{n}-x_{n+1}\right\| .
$$

By (3.14) and (3.16), we have

$$
\lim _{n \rightarrow \infty}\left\|y_{n, i}-x_{n}\right\|=0, \quad i=1,2, \ldots
$$

Step 3. We claim that the following statements hold:

(S1) $\lim _{n \rightarrow \infty}\left\|x_{n}-u_{n}\right\|=0$,

(S2) $\lim _{n \rightarrow \infty}\left\|x_{n}-t_{n}\right\|=0$,

(S3) $\lim _{n \rightarrow \infty}\left\|w_{n}-x_{n}\right\|=0$.

For (3.2), we note that

$$
\begin{aligned}
\left\|y_{n, i}-p\right\|^{2} \leq & \alpha_{n, i}\left\|x_{0}-p\right\|^{2}+\left(1-\alpha_{n, i}\right)\left\|T_{i} w_{n}-p\right\|^{2} \\
= & \alpha_{n, i}\left\|x_{0}-p\right\|^{2}+\left(1-\alpha_{n, i}\right)\left\|w_{n}-p\right\|^{2} \\
\leq & \alpha_{n, i}\left\|x_{0}-p\right\|^{2}+\left(1-\alpha_{n, i}\right) \\
& \times\left\{\xi_{n}\left\|\left(x_{n}-s_{n} B x_{n}\right)-\left(p-s_{n} B p\right)\right\|^{2}+\left(1-\xi_{n}\right)\left\|\left(x_{n}-r_{n} A x_{n}\right)-\left(p-r_{n} A p\right)\right\|^{2}\right\} \\
\leq & \alpha_{n, i}\left\|x_{0}-p\right\|^{2}+\left(1-\alpha_{n, i}\right) \\
& \times\left\{\xi_{n}\left(\left\|x_{n}-p\right\|^{2}+s_{n}\left(s_{n}-2 \beta\right)\left\|B x_{n}-B p\right\|^{2}\right)\right. \\
& \left.+\left(1-\xi_{n}\right)\left(\left\|x_{n}-p\right\|^{2}+r_{n}\left(r_{n}-2 \alpha\right)\left\|A x_{n}-A p\right\|^{2}\right)\right\} \\
= & \alpha_{n, i}\left\|x_{0}-p\right\|^{2}+\left(1-\alpha_{n, i}\right) \\
& \times\left\{\left\|x_{n}-p\right\|^{2}+\xi_{n} s_{n}\left(s_{n}-2 \beta\right)\left\|B x_{n}-B p\right\|^{2}+\left(1-\xi_{n}\right) r_{n}\left(r_{n}-2 \alpha\right)\left\|A x_{n}-A p\right\|^{2}\right\} \\
= & \alpha_{n, i}\left\|x_{0}-p\right\|^{2}+\left\|x_{n}-p\right\|^{2}+\left(1-\alpha_{n, i}\right) \xi_{n} s_{n}\left(s_{n}-2 \beta\right)\left\|B x_{n}-B p\right\|^{2} \\
& +\left(1-\alpha_{n, i}\right)\left(1-\xi_{n}\right) r_{n}\left(r_{n}-2 \alpha\right)\left\|A x_{n}-A p\right\|^{2} \\
= & \alpha_{n, i}\left\|x_{0}-p\right\|^{2}+\left\|x_{n}-p\right\|^{2}+\left(1-\alpha_{n, i}\right) \xi_{n} s_{n}\left(s_{n}-2 \beta\right)\left\|B x_{n}-B p\right\|^{2} .
\end{aligned}
$$

Since $0<c \leq s_{n} \leq d \leq 2 \beta, 0 \leq k_{i} \leq \alpha_{n, i} \leq h_{i}<1$, we have

$$
\begin{aligned}
\left(1-h_{i}\right) \xi c(2 \beta-d)\left\|B x_{n}-B p\right\|^{2} & \leq \alpha_{n, i}\left\|x_{0}-p\right\|^{2}+\left\|x_{n}-p\right\|^{2}-\left\|y_{n, i}-p\right\|^{2} \\
& \leq \alpha_{n, i}\left\|x_{0}-p\right\|^{2}+\left\|y_{n, i}-x_{n}\right\|\left(\left\|x_{n}-p\right\|+\left\|y_{n, i}-p\right\|\right) .
\end{aligned}
$$


By condition (iii) and (3.18), $\lim _{n \rightarrow \infty}\left\|B x_{n}-B p\right\|=0$ by using the same method with (3.20). Hence, from (3.19), since $0<a \leq r_{n} \leq b \leq 2 \alpha, 0 \leq k_{i} \leq \alpha_{n, i} \leq h_{i}<1$, we have

$$
\begin{aligned}
\left(1-h_{i}\right)(1-\xi) a(2 \alpha-b)\left\|A x_{n}-A p\right\|^{2} & \leq \alpha_{n, i}\left\|x_{0}-p\right\|^{2}+\left\|x_{n}-p\right\|^{2}-\left\|y_{n, i}-p\right\|^{2} \\
& \leq \alpha_{n, i}\left\|x_{0}-p\right\|^{2}+\left\|y_{n, i}-x_{n}\right\|\left(\left\|x_{n}-p\right\|+\left\|y_{n, i}-p\right\|\right) .
\end{aligned}
$$

By condition (iii) and (3.18), then we have $\lim _{n \rightarrow \infty}\left\|A x_{n}-A p\right\|=0$. On the other hand, we compute

$$
\begin{aligned}
\left\|u_{n}-p\right\|^{2}= & \left\|T_{s_{n}}^{\left(F_{2}, \varphi_{2}\right)}\left(I-s_{n} B\right) x_{n}-T_{s_{n}}^{\left(F_{2}, \varphi_{2}\right)}\left(I-s_{n} B\right) p\right\|^{2} \\
\leq & \left\langle\left(x_{n}-s_{n} B x_{n}\right)-\left(p-s_{n} B p\right), u_{n}-p\right\rangle \\
= & \frac{1}{2}\left\{\left\|\left(x_{n}-s_{n} B x_{n}\right)-\left(p-s_{n} B p\right)\right\|^{2}+\left\|u_{n}-p\right\|^{2}\right. \\
& \left.\quad-\left\|\left(x_{n}-s_{n} B x_{n}\right)-\left(p-s_{n} B p\right)-\left(u_{n}-p\right)\right\|^{2}\right\} \\
\leq & \frac{1}{2}\left\{\left\|x_{n}-p\right\|^{2}+\left\|u_{n}-p\right\|^{2}-\left\|\left(x_{n}-s_{n} B x_{n}\right)-\left(p-s_{n} B p\right)-\left(u_{n}-p\right)\right\|^{2}\right\} \\
= & \frac{1}{2}\left\{\left\|x_{n}-p\right\|^{2}+\left\|u_{n}-p\right\|^{2}-\left\|u_{n}-x_{n}\right\|^{2}+2 s_{n}\left\langle x_{n}-u_{n}, B x_{n}-B p\right\rangle\right. \\
& \left.\quad-s_{n}^{2}\left\|B x_{n}-B p\right\|^{2}\right\},
\end{aligned}
$$

and hence,

$$
\begin{aligned}
\left\|u_{n}-p\right\|^{2} & \leq\left\|x_{n}-p\right\|^{2}-\left\|u_{n}-x_{n}\right\|^{2}+2 s_{n}\left\langle x_{n}-u_{n}, B x_{n}-B p\right\rangle-s_{n}^{2}\left\|B x_{n}-B p\right\|^{2} \\
& \leq\left\|x_{n}-p\right\|^{2}-\left\|u_{n}-x_{n}\right\|^{2}+2 s_{n}\left\|x_{n}-u_{n}\right\|\left\|B x_{n}-B p\right\| .
\end{aligned}
$$

By using the same method as (3.23), we also have

$$
\begin{aligned}
\left\|t_{n}-p\right\|^{2} & \leq\left\|x_{n}-p\right\|^{2}-\left\|t_{n}-x_{n}\right\|^{2}+2 r_{n}\left\langle x_{n}-t_{n}, A x_{n}-A p\right\rangle-r_{n}^{2}\left\|A x_{n}-A p\right\|^{2} \\
& \leq\left\|x_{n}-p\right\|^{2}-\left\|t_{n}-x_{n}\right\|^{2}+2 r_{n}\left\|x_{n}-t_{n}\right\|\left\|A x_{n}-A p\right\| .
\end{aligned}
$$


Furthermore, we observe that

$$
\begin{aligned}
& \left\|y_{n, i}-p\right\|^{2} \\
& \leq \alpha_{n, i}\left\|x_{0}-p\right\|^{2}+\left(1-\alpha_{n, i}\right)\left\|T_{i} w_{n}-p\right\|^{2} \\
& =\alpha_{n, i}\left\|x_{0}-p\right\|^{2}+\left(1-\alpha_{n, i}\right)\left\|w_{n}-p\right\|^{2} \\
& \leq \alpha_{n, i}\left\|x_{0}-p\right\|^{2}+\left(1-\alpha_{n, i}\right)\left\{\left\|\xi_{n} P_{C}\left(u_{n}-\lambda_{n} D u_{n}\right)+\left(1-\xi_{n}\right) P_{C}\left(t_{n}-\mu_{n} E t_{n}\right)-p\right\|^{2}\right\} \\
& \leq \alpha_{n, i}\left\|x_{0}-p\right\|^{2}+\left(1-\alpha_{n, i}\right) \\
& \times\left\{\xi_{n}\left(\left\|u_{n}-p\right\|^{2}-\lambda_{n}\left(2 \delta-\lambda_{n}\right)\left\|D u_{n}-D p\right\|^{2}\right)\right. \\
& \left.+\left(1-\xi_{n}\right)\left(\left\|t_{n}-p\right\|^{2}-\mu_{n}\left(2 \eta-\mu_{n}\right)\left\|E t_{n}-E p\right\|^{2}\right)\right\} \\
& \leq \alpha_{n, i}\left\|x_{0}-p\right\|^{2}+\left(1-\alpha_{n, i}\right)\left\{\xi_{n}\left\|u_{n}-p\right\|^{2}+\left(1-\xi_{n}\right)\left\|t_{n}-p\right\|^{2}\right\} \\
& \leq \alpha_{n, i}\left\|x_{0}-p\right\|^{2}+\left(1-\alpha_{n, i}\right) \\
& \times\left\{\xi_{n}\left(\left\|x_{n}-p\right\|^{2}-\left\|u_{n}-x_{n}\right\|^{2}+2 s_{n}\left\|x_{n}-u_{n}\right\|\left\|B x_{n}-B p\right\|\right)\right. \\
& \left.+\left(1-\xi_{n}\right)\left(\left\|x_{n}-p\right\|^{2}-\left\|t_{n}-x_{n}\right\|^{2}+2 r_{n}\left\|x_{n}-t_{n}\right\|\left\|A x_{n}-A p\right\|\right)\right\} \\
& \leq \alpha_{n, i}\left\|x_{0}-p\right\|^{2}+\left\|x_{n}-p\right\|^{2}-\left(1-\alpha_{n, i}\right) \xi_{n}\left\|u_{n}-x_{n}\right\|^{2} \\
& +\left(1-\alpha_{n, i}\right) \xi_{n} 2 s_{n}\left\|x_{n}-u_{n}\right\|\left\|B x_{n}-B p\right\|-\left(1-\alpha_{n, i}\right)\left(1-\xi_{n}\right)\left\|t_{n}-x_{n}\right\|^{2} \\
& +\left(1-\alpha_{n, i}\right)\left(1-\xi_{n}\right) 2 r_{n}\left\|x_{n}-t_{n}\right\|\left\|A x_{n}-A p\right\| \\
& \leq \alpha_{n, i}\left\|x_{0}-p\right\|^{2}+\left\|x_{n}-p\right\|^{2}-\left(1-\alpha_{n, i}\right) \xi_{n}\left\|u_{n}-x_{n}\right\|^{2}+\left(1-\alpha_{n, i}\right) \xi_{n} 2 s_{n}\left\|x_{n}-u_{n}\right\|\left\|B x_{n}-B p\right\| \\
& +\left(1-\alpha_{n, i}\right)\left(1-\xi_{n}\right) 2 r_{n}\left\|x_{n}-t_{n}\right\|\left\|A x_{n}-A p\right\| \text {. }
\end{aligned}
$$

By condition (i)-(iv), (3.18), $\lim _{n \rightarrow \infty}\left\|A x_{n}-A p\right\|=0$ and $\lim _{n \rightarrow \infty}\left\|B x_{n}-B p\right\|=0$, then we get

$$
\begin{aligned}
\left(1-\alpha_{n, i}\right) \xi_{n}\left\|u_{n}-x_{n}\right\|^{2} \leq & \alpha_{n, i}\left\|x_{0}-p\right\|^{2}+\left\|x_{n}-p\right\|^{2}-\left\|y_{n, i}-p\right\|^{2} \\
& +\left(1-\alpha_{n, i}\right) \xi_{n} 2 s_{n}\left\|x_{n}-u_{n}\right\|\left\|B x_{n}-B p\right\| \\
& +\left(1-\alpha_{n, i}\right)\left(1-\xi_{n}\right) 2 r_{n}\left\|x_{n}-t_{n}\right\|\left\|A x_{n}-A p\right\| \\
\leq & \alpha_{n, i}\left\|x_{0}-p\right\|^{2}+\left\|x_{n}-y_{n, i}\right\|\left(\left\|x_{n}-p\right\|+\left\|y_{n, i}-p\right\|\right) \\
& +\left(1-\alpha_{n, i}\right) \xi_{n} 2 s_{n}\left\|x_{n}-u_{n}\right\|\left\|B x_{n}-B p\right\| \\
& +\left(1-\alpha_{n, i}\right)\left(1-\xi_{n}\right) 2 r_{n}\left\|x_{n}-t_{n}\right\|\left\|A x_{n}-A p\right\| .
\end{aligned}
$$


Therefore, we have

$$
\lim _{n \rightarrow \infty}\left\|x_{n}-u_{n}\right\|=0
$$

Similar to (3.26), from (3.25) by conditions (i)-(iv), (3.18), $\lim _{n \rightarrow \infty}\left\|A x_{n}-A p\right\|=0$, and $\lim _{n \rightarrow \infty}\left\|B x_{n}-B p\right\|=0$, we get

$$
\begin{aligned}
\left(1-\alpha_{n, i}\right)\left(1-\xi_{n}\right)\left\|t_{n}-x_{n}\right\|^{2} \leq & \alpha_{n, i}\left\|x_{0}-p\right\|^{2}+\left\|x_{n}-p\right\|^{2}-\left\|y_{n, i}-p\right\|^{2} \\
& +\left(1-\alpha_{n, i}\right) \xi_{n} 2 s_{n}\left\|x_{n}-u_{n}\right\|\left\|B x_{n}-B p\right\| \\
& +\left(1-\alpha_{n, i}\right)\left(1-\xi_{n}\right) 2 r_{n}\left\|x_{n}-t_{n}\right\|\left\|A x_{n}-A p\right\| \\
\leq & \alpha_{n, i}\left\|x_{0}-p\right\|^{2}+\left\|x_{n}-y_{n, i}\right\|\left(\left\|x_{n}-p\right\|+\left\|y_{n, i}-p\right\|\right) \\
& +\left(1-\alpha_{n, i}\right) \xi_{n} 2 s_{n}\left\|x_{n}-u_{n}\right\|\left\|B x_{n}-B p\right\| \\
& +\left(1-\alpha_{n, i}\right)\left(1-\xi_{n}\right) 2 r_{n}\left\|x_{n}-t_{n}\right\|\left\|A x_{n}-A p\right\| .
\end{aligned}
$$

Therefore, we have

$$
\lim _{n \rightarrow \infty}\left\|x_{n}-t_{n}\right\|=0
$$

From (3.1), (3.3), we have

$$
\begin{aligned}
& \left\|w_{n}-p\right\|^{2}=\| \xi_{n} P_{C}\left(u_{n}-\lambda_{n} D u_{n}\right)+\left(1-\xi_{n}\right) P_{C}\left(t_{n}-\mu_{n} E t_{n}\right) \\
& -\xi_{n} P_{C}\left(p-\lambda_{n} D p\right)-\left(1-\xi_{n}\right) P_{C}\left(p-\mu_{n} E p\right) \|^{2} \\
& =\xi_{n}\left\|P_{C}\left(u_{n}-\lambda_{n} D u_{n}\right)-P_{C}\left(p-\lambda_{n} D p\right)\right\|^{2} \\
& +\left(1-\xi_{n}\right)\left\|P_{C}\left(t_{n}-\mu_{n} E t_{n}\right)-P_{C}\left(p-\mu_{n} E p\right)\right\|^{2} \\
& \leq \xi_{n}\left\{\left\|u_{n}-p\right\|^{2}-\lambda_{n}\left(2 \delta-\lambda_{n}\right)\left\|D u_{n}-D p\right\|^{2}\right\} \\
& +\left(1-\xi_{n}\right)\left\{\left\|t_{n}-p\right\|^{2}-\mu_{n}\left(2 \eta-\mu_{n}\right)\left\|E t_{n}-E p\right\|^{2}\right\} \\
& \leq \xi_{n}\left\{\left\|x_{n}-p\right\|^{2}+s_{n}\left(s_{n}-2 \beta\right)\left\|B x_{n}-B p\right\|^{2}-\lambda_{n}\left(2 \delta-\lambda_{n}\right)\left\|D u_{n}-D p\right\|^{2}\right\} \\
& +\left(1-\xi_{n}\right)\left\{\left\|x_{n}-p\right\|^{2}+r_{n}\left(r_{n}-2 \alpha\right)\left\|A x_{n}-A p\right\|^{2}-\mu_{n}\left(2 \eta-\mu_{n}\right)\left\|E t_{n}-E p\right\|^{2}\right\} \\
& \leq\left\|x_{n}-p\right\|^{2}+\xi_{n} s_{n}\left(s_{n}-2 \beta\right)\left\|B x_{n}-B p\right\|^{2}-\xi_{n} \lambda_{n}\left(2 \delta-\lambda_{n}\right)\left\|D u_{n}-D p\right\|^{2} \\
& +\left(1-\xi_{n}\right) r_{n}\left(r_{n}-2 \alpha\right)\left\|A x_{n}-A p\right\|^{2}-\left(1-\xi_{n}\right) \mu_{n}\left(2 \eta-\mu_{n}\right)\left\|E t_{n}-E p\right\|^{2} .
\end{aligned}
$$


Furthermore, we observe that

$$
\begin{aligned}
\left\|y_{n, i}-p\right\|^{2} \leq & \alpha_{n, i}\left\|x_{0}-p\right\|^{2}+\left(1-\alpha_{n, i}\right)\left\|T_{i} w_{n}-p\right\|^{2} \\
= & \alpha_{n, i}\left\|x_{0}-p\right\|^{2}+\left(1-\alpha_{n, i}\right)\left\|w_{n}-p\right\|^{2} \\
\leq & \alpha_{n, i}\left\|x_{0}-p\right\|^{2}+\left(1-\alpha_{n, i}\right) \\
& \times\left\{\left\|x_{n}-p\right\|^{2}+\xi_{n} s_{n}\left(s_{n}-2 \beta\right)\left\|B x_{n}-B p\right\|^{2}-\xi_{n} \lambda_{n}\left(2 \delta-\lambda_{n}\right)\left\|D u_{n}-D p\right\|^{2}\right. \\
& \left.+\left(1-\xi_{n}\right) r_{n}\left(r_{n}-2 \alpha\right)\left\|A x_{n}-A p\right\|^{2}-\left(1-\xi_{n}\right) \mu_{n}\left(2 \eta-\mu_{n}\right)\left\|E t_{n}-E p\right\|^{2}\right\} \\
\leq & \alpha_{n, i}\left\|x_{0}-p\right\|^{2}+\left\|x_{n}-p\right\|^{2}+\left(1-\alpha_{n, i}\right) \xi_{n} s_{n}\left(s_{n}-2 \beta\right)\left\|B x_{n}-B p\right\|^{2}-\left(1-\alpha_{n, i}\right) \xi_{n} \lambda_{n} \\
& \times\left(2 \delta-\lambda_{n}\right)\left\|D u_{n}-D p\right\|^{2}+\left(1-\alpha_{n, i}\right)\left(1-\xi_{n}\right) r_{n}\left(r_{n}-2 \alpha\right)\left\|A x_{n}-A p\right\|^{2} \\
& -\left(1-\alpha_{n, i}\right)\left(1-\xi_{n}\right) \mu_{n}\left(2 \eta-\mu_{n}\right)\left\|E t_{n}-E p\right\|^{2} \\
\leq & \alpha_{n, i}\left\|x_{0}-p\right\|^{2}+\left\|x_{n}-p\right\|^{2}+\left(1-\alpha_{n, i}\right) \xi_{n} s_{n}\left(s_{n}-2 \beta\right)\left\|B x_{n}-B p\right\|^{2} \\
& -\left(1-\alpha_{n, i}\right) \xi_{n} \lambda_{n}\left(2 \delta-\lambda_{n}\right)\left\|D u_{n}-D p\right\|^{2} \\
& +\left(1-\alpha_{n, i}\right)\left(1-\xi_{n}\right) r_{n}\left(r_{n}-2 \alpha\right)\left\|A x_{n}-A p\right\|^{2} .
\end{aligned}
$$

Since $0<e \leq \lambda_{n} \leq f<2 \delta, 0 \leq k_{i} \leq \alpha_{n, i} \leq h_{i}<1$, we have

$$
\begin{aligned}
\left(1-h_{i}\right) \xi e(2 \delta-f)\left\|D u_{n}-D p\right\|^{2} \leq & \alpha_{n, i}\left\|x_{0}-p\right\|^{2}+\left\|x_{n}-p\right\|^{2}-\left\|y_{n, i}-p\right\|^{2} \\
& +\left(1-\alpha_{n, i}\right) \xi_{n} s_{n}\left(s_{n}-2 \beta\right)\left\|B x_{n}-B p\right\|^{2} \\
& +\left(1-\alpha_{n, i}\right)\left(1-\xi_{n}\right) r_{n}\left(r_{n}-2 \alpha\right)\left\|A x_{n}-A p\right\|^{2} \\
\leq & \alpha_{n, i}\left\|x_{0}-p\right\|^{2}+\left\|y_{n, i}-x_{n}\right\|\left(\left\|x_{n}-p\right\|-\left\|y_{n, i}-p\right\|\right) \\
& +\left(1-\alpha_{n, i}\right) \xi_{n} s_{n}\left(s_{n}-2 \beta\right)\left\|B x_{n}-B p\right\|^{2} \\
& +\left(1-\alpha_{n, i}\right)\left(1-\xi_{n}\right) r_{n}\left(r_{n}-2 \alpha\right)\left\|A x_{n}-A p\right\|^{2} .
\end{aligned}
$$


By conditions (i) $-(\mathrm{v}),(3.18), \lim _{n \rightarrow \infty}\left\|A x_{n}-A p\right\|=0$, and $\lim _{n \rightarrow \infty}\left\|B x_{n}-B p\right\|=0$, then $\lim _{n \rightarrow \infty}\left\|D u_{n}-D p\right\|=0$. By using the same method with (3.32). Hence, from (3.31), and since $0<g \leq \mu_{n} \leq j \leq 2 \eta, 0 \leq k_{i} \leq \alpha_{n, i} \leq h_{i} \leq 1$, we have

$$
\begin{aligned}
\left(1-h_{i}\right) & (1-\xi) g(2 \eta-j)\left\|E t_{n}-E p\right\|^{2} \\
\leq & \alpha_{n, i}\left\|x_{0}-p\right\|^{2}+\left\|x_{n}-p\right\|^{2}-\left\|y_{n, i}-p\right\|^{2} \\
& +\left(1-\alpha_{n, i}\right) \xi_{n} s_{n}\left(s_{n}-2 \beta\right)\left\|B x_{n}-B p\right\|^{2} \\
& +\left(1-\alpha_{n, i}\right)\left(1-\xi_{n}\right) r_{n}\left(r_{n}-2 \alpha\right)\left\|A x_{n}-A p\right\|^{2} \\
\leq & \alpha_{n, i}\left\|x_{0}-p\right\|^{2}+\left\|y_{n, i}-x_{n}\right\|\left(\left\|x_{n}-p\right\|-\left\|y_{n, i}-p\right\|\right) \\
& +\left(1-\alpha_{n, i}\right) \xi_{n} s_{n}\left(s_{n}-2 \beta\right)\left\|B x_{n}-B p\right\|^{2} \\
& +\left(1-\alpha_{n, i}\right)\left(1-\xi_{n}\right) r_{n}\left(r_{n}-2 \alpha\right)\left\|A x_{n}-A p\right\|^{2} .
\end{aligned}
$$

By conditions (i)-(iv), (vi), (3.18), $\lim _{n \rightarrow \infty}\left\|A x_{n}-A p\right\|=0$, and $\lim _{n \rightarrow \infty}\left\|B x_{n}-B p\right\|=0$, then $\lim _{n \rightarrow \infty}\left\|E t_{n}-E p\right\|=0$. From (3.1), we have

$$
\begin{aligned}
\left\|w_{n}-p\right\|^{2} \leq & \| \xi_{n}\left\{P_{C}\left(u_{n}-\lambda_{n} D u_{n}\right)-P_{C}\left(p-\lambda_{n} D p\right)\right\} \\
& +\left(1-\xi_{n}\right)\left\{P_{C}\left(t_{n}-\mu_{n} E t_{n}\right)-P_{C}\left(p-\mu_{n} E p\right)\right\} \|^{2} \\
\leq & \xi_{n}\left\|u_{n}^{\prime}-p\right\|^{2}+\left(1-\xi_{n}\right)\left\|t_{n}^{\prime}-p\right\|^{2} .
\end{aligned}
$$

Assume that $u_{n}^{\prime}=P_{C}\left(u_{n}-\lambda_{n} D u_{n}\right)$ and $t_{n}^{\prime}=P_{C}\left(t_{n}-\mu_{n} E t_{n}\right)$. By nonexpansiveness of $I-\lambda_{n} D$ and $I-\mu_{n} E$, we also have

$$
\begin{aligned}
\left\|u_{n}^{\prime}-p\right\|^{2} \leq & \left\|P_{C}\left(I-\lambda_{n} D\right) u_{n}-P_{C}\left(I-\lambda_{n} D\right) p\right\|^{2} \\
\leq & \left\langle\left(u_{n}-\lambda_{n} D u_{n}\right)-\left(p-\lambda_{n} D p\right), u_{n}^{\prime}-p\right\rangle \\
= & \frac{1}{2}\left\{\left\|\left(u_{n}-\lambda_{n} D u_{n}\right)-\left(p-\lambda_{n} D p\right)\right\|^{2}+\left\|u_{n}^{\prime}-p\right\|^{2}\right. \\
& \left.\quad-\left\|\left(u_{n}-\lambda_{n} D u_{n}\right)-\left(p-\lambda_{n} D p\right)-\left(u_{n}^{\prime}-p\right)\right\|^{2}\right\} \\
\leq & \frac{1}{2}\left\{\left\|u_{n}-p\right\|^{2}+\left\|u_{n}^{\prime}-p\right\|^{2}-\left\|\left(u_{n}-\lambda_{n} D u_{n}\right)-\left(p-\lambda_{n} D p\right)-\left(u_{n}^{\prime}-p\right)\right\|^{2}\right\} \\
= & \frac{1}{2}\left\{\left\|T_{\left.s_{n}, F_{2}\right)}\left(x_{n}-s_{n} B x_{n}\right)-T_{s_{n}}^{\left(F_{2}, \varphi_{2}\right)}\left(p-s_{n} B p\right)\right\|^{2}+\left\|u_{n}^{\prime}-p\right\|^{2}-\left\|u_{n}-u_{n}^{\prime}\right\|^{2}\right. \\
& \left.\quad+2 \lambda_{n}\left\langle u_{n}-u_{n}^{\prime}, D u_{n}-D p\right\rangle-\lambda_{n}^{2}\left\|D u_{n}-D p\right\|^{2}\right\} \\
\leq & \frac{1}{2}\left\{\left\|x_{n}-p\right\|^{2}+\left\|u_{n}^{\prime}-p\right\|^{2}-\left\|u_{n}-u_{n}^{\prime}\right\|^{2}+\lambda_{n}\left(\lambda_{n}-2 \delta\right)\left\|D u_{n}-D p\right\|^{2}\right\} .
\end{aligned}
$$


It follows that

$$
\left\|u_{n}^{\prime}-p\right\|^{2} \leq\left\|x_{n}-p\right\|^{2}-\left\|u_{n}-u_{n}^{\prime}\right\|^{2}+\lambda_{n}\left(\lambda_{n}-2 \delta\right)\left\|D u_{n}-D p\right\|^{2}
$$

Similar to (3.36), we obtain

$$
\left\|t_{n}^{\prime}-p\right\|^{2} \leq\left\|x_{n}-p\right\|^{2}-\left\|t_{n}-t_{n}^{\prime}\right\|^{2}+\mu_{n}\left(\mu_{n}-2 \eta\right)\left\|E t_{n}-E p\right\|^{2} .
$$

Substituting (3.36), (3.37) into (3.34), we have

$$
\begin{aligned}
\left\|w_{n}-p\right\|^{2} \leq & \xi_{n}\left\|u_{n}^{\prime}-p\right\|^{2}+\left(1-\xi_{n}\right)\left\|t_{n}^{\prime}-p\right\|^{2} \\
\leq & \xi_{n}\left\{\left\|x_{n}-p\right\|^{2}-\left\|u_{n}-u_{n}^{\prime}\right\|^{2}+\lambda_{n}\left(\lambda_{n}-2 \delta\right)\left\|D u_{n}-D p\right\|^{2}\right\} \\
& +\left(1-\xi_{n}\right)\left\{\left\|x_{n}-p\right\|^{2}-\left\|t_{n}-t_{n}^{\prime}\right\|^{2}+\mu_{n}\left(\mu_{n}-2 \eta\right)\left\|E t_{n}-E p\right\|^{2}\right\} \\
\leq & \left\|x_{n}-p\right\|^{2}-\xi_{n}\left\|u_{n}-u_{n}^{\prime}\right\|^{2}+\xi_{n} \lambda_{n}\left(\lambda_{n}-2 \delta\right)\left\|D u_{n}-D p\right\|^{2} \\
& -\left(1-\xi_{n}\right)\left\|t_{n}-t_{n}^{\prime}\right\|^{2}+\left(1-\xi_{n}\right) \mu_{n}\left(\mu_{n}-2 \eta\right)\left\|E t_{n}-E p\right\|^{2} .
\end{aligned}
$$

By (3.38), we have

$$
\begin{aligned}
&\left\|y_{n, i}-p\right\|^{2} \\
& \leq \alpha_{n, i}\left\|x_{0}-p\right\|^{2}+\left(1-\alpha_{n, i}\right)\left\|T_{i} w_{n}-p\right\|^{2} \\
&= \alpha_{n, i}\left\|x_{0}-p\right\|^{2}+\left(1-\alpha_{n, i}\right)\left\|w_{n}-p\right\|^{2} \\
&= \alpha_{n, i}\left\|x_{0}-p\right\|^{2}+\left(1-\alpha_{n, i}\right) \\
& \times\left\{\left\|x_{n}-p\right\|^{2}-\xi_{n}\left\|u_{n}-u_{n}^{\prime}\right\|^{2}+\xi_{n} \lambda_{n}\left(\lambda_{n}-2 \delta\right)\left\|D u_{n}-D p\right\|^{2}\right. \\
&\left.\quad-\left(1-\xi_{n}\right)\left\|t_{n}-t_{n}^{\prime}\right\|^{2}+\left(1-\xi_{n}\right) \mu_{n}\left(\mu_{n}-2 \eta\right)\left\|E t_{n}-E p\right\|^{2}\right\} \\
&= \alpha_{n, i}\left\|x_{0}-p\right\|^{2}+\left\|x_{n}-p\right\|^{2}-\left(1-\alpha_{n, i}\right) \xi_{n}\left\|u_{n}-u_{n}^{\prime}\right\|^{2} \\
&+\left(1-\alpha_{n, i}\right) \xi_{n} \lambda_{n}\left(\lambda_{n}-2 \delta\right)\left\|D u_{n}-D p\right\|^{2}-\left(1-\alpha_{n, i}\right)\left(1-\xi_{n}\right)\left\|t_{n}-t_{n}^{\prime}\right\|^{2} \\
&+\left(1-\alpha_{n, i}\right)\left(1-\xi_{n}\right) \mu_{n}\left(\mu_{n}-2 \eta\right)\left\|E t_{n}-E p\right\|^{2} \\
&= \alpha_{n, i}\left\|x_{0}-p\right\|^{2}+\left\|x_{n}-p\right\|^{2}-\left(1-\alpha_{n, i}\right) \xi_{n}\left\|u_{n}-u_{n}^{\prime}\right\|^{2} \\
&+\left(1-\alpha_{n, i}\right) \xi_{n} \lambda_{n}\left(\lambda_{n}-2 \delta\right)\left\|D u_{n}-D p\right\|^{2}+\left(1-\alpha_{n, i}\right)\left(1-\xi_{n}\right) \mu_{n}\left(\mu_{n}-2 \eta\right)\left\|E t_{n}-E p\right\|^{2} .
\end{aligned}
$$


It follows that

$$
\begin{aligned}
\left(1-\alpha_{n, i}\right) \xi_{n}\left\|u_{n}-u_{n}^{\prime}\right\|^{2} \leq & \alpha_{n, i}\left\|x_{0}-p\right\|^{2}+\left\|x_{n}-p\right\|^{2}-\left\|y_{n, i}-p\right\|^{2}+\left(1-\alpha_{n, i}\right) \xi_{n} \lambda_{n} \\
& \times\left(\lambda_{n}-2 \delta\right)\left\|D u_{n}-D p\right\|^{2}+\left(1-\alpha_{n, i}\right)\left(1-\xi_{n}\right) \mu_{n}\left(\mu_{n}-2 \eta\right)\left\|E t_{n}-E p\right\|^{2} \\
\leq & \alpha_{n, i}\left\|x_{0}-p\right\|^{2}+\left\|x_{n}-y_{n, i}\right\|\left(\left\|x_{n}-p\right\|+\left\|y_{n, i}-p\right\|\right)+\left(1-\alpha_{n, i}\right) \xi_{n} \lambda_{n} \\
& \times\left(\lambda_{n}-2 \delta\right)\left\|D u_{n}-D p\right\|^{2}+\left(1-\alpha_{n, i}\right)\left(1-\xi_{n}\right) \mu_{n}\left(\mu_{n}-2 \eta\right)\left\|E t_{n}-E p\right\|^{2} .
\end{aligned}
$$

By conditions (iii)-(vi), (3.18), $\lim _{n \rightarrow \infty}\left\|D u_{n}-D p\right\|=0$ and $\lim _{n \rightarrow \infty}\left\|E t_{n}-E p\right\|=0$, we get

$$
\lim _{n \rightarrow \infty}\left\|u_{n}-u_{n}^{\prime}\right\|=0
$$

By using (3.41), we can prove that

$$
\lim _{n \rightarrow \infty}\left\|t_{n}-t_{n}^{\prime}\right\|=0
$$

Applying (3.27) and (3.41), we also have

$$
\lim _{n \rightarrow \infty}\left\|x_{n}-u_{n}^{\prime}\right\|=0
$$

From (3.29) and (3.42), we obtain

$$
\lim _{n \rightarrow \infty}\left\|x_{n}-t_{n}^{\prime}\right\|=0
$$

Since $u_{n}^{\prime}=P_{C}\left(u_{n}-\lambda_{n} D u_{n}\right)$ and $t_{n}^{\prime}=P_{C}\left(t_{n}-\mu_{n} E t_{n}\right)$, we have

$$
w_{n}-x_{n}=\xi_{n}\left(u_{n}^{\prime}-x_{n}\right)+\left(1-\xi_{n}\right)\left(t_{n}^{\prime}-x_{n}\right) .
$$

By (3.43) and (3.44), we obtain

$$
\lim _{n \rightarrow \infty}\left\|w_{n}-x_{n}\right\|=0
$$

By condition (iii), we have $y_{n, i}=\alpha_{n, i} x_{0}+\left(1-\alpha_{n, i}\right) T_{i} w_{n}$, which implies that

$$
\left\|y_{n, i}-T_{i} w_{n}\right\|=\alpha_{n, i}\left\|x_{0}-T_{i} w_{n}\right\| \longrightarrow 0, \quad n \longrightarrow \infty, \forall i \geq 1 .
$$

From (3.18) and $\lim _{n \rightarrow \infty}\left\|y_{n, i}-T_{i} w_{n}\right\|=0$, we have

$$
\left\|x_{n}-T_{i} w_{n}\right\| \leq\left\|y_{n, i}-T_{i} w_{n}\right\|+\left\|y_{n, i}-x_{n}\right\| \longrightarrow 0, \quad n \longrightarrow \infty, \forall i \geq 1
$$


Since

$$
\left\|w_{n}-T_{i} w_{n}\right\| \leq\left\|w_{n}-x_{n}\right\|+\left\|x_{n}-T_{i} w_{n}\right\|
$$

By (3.46) and (3.48), we have that $\lim _{n \rightarrow \infty}\left\|w_{n}-T_{i} w_{n}\right\|=0$, for all $i=1,2, \ldots$.

Step 4. We show that $z \in \Theta:=\left(\bigcap_{i=1}^{\infty} F\left(T_{i}\right)\right) \cap \operatorname{GMEP}\left(F_{1}, \varphi_{1}, A\right) \cap \operatorname{GMEP}\left(F_{2}, \varphi_{2}, B\right) \cap \operatorname{VI}(C, D) \cap$ $\operatorname{VI}(C, E)$.

First, we show that $z \in \bigcap_{i=1}^{\infty} F\left(T_{i}\right)$. Assume that $z \notin \bigcap_{i=1}^{\infty} F\left(T_{i}\right)$. Since $\lim _{n \rightarrow \infty} \| w_{n}-$ $x_{n} \|=0$ and $\lim _{n \rightarrow \infty}\left\|x_{n}-z\right\|=0$, we have that $\lim _{n \rightarrow \infty}\left\|w_{n}-z\right\|=0$. By $\lim _{n \rightarrow \infty}\left\|w_{n}-z\right\|=0$ and $\lim _{n \rightarrow \infty}\left\|w_{n}-T_{i} w_{n}\right\|=0, i=1,2, \ldots$, from Opial's condition, we have

$$
\begin{aligned}
\liminf _{i \rightarrow \infty}\left\|w_{n_{i}}-z\right\| & <\liminf _{i \rightarrow \infty}\left\|w_{n_{i}}-T_{i} z\right\| \\
& \leq \liminf _{i \rightarrow \infty}\left(\left\|w_{n_{i}}-T_{i} w_{n_{i}}\right\|+\left\|T_{i} w_{n_{i}}-T_{i} z\right\|\right) \\
& \leq \liminf _{i \rightarrow \infty}\left\|w_{n_{i}}-z\right\|,
\end{aligned}
$$

which is a contradiction. Thus, we obtain $z \in \bigcap_{i=1}^{\infty} F\left(T_{i}\right)$.

Next, we show that $z \in \operatorname{GMEP}\left(F_{1}, \varphi, A\right)$. Since $t_{n}=T_{r_{n}}^{\left(F_{1}, \varphi_{1}\right)}\left(x_{n}-r_{n} A x_{n}\right), n \geq 1$, we have for any $y \in C$ that

$$
F_{1}\left(t_{n}, y\right)+\varphi_{1}(y)-\varphi_{1}\left(t_{n}\right)+\left\langle A x_{n}, y-t_{n}\right\rangle+\frac{1}{r_{n}}\left\langle y-t_{n}, t_{n}-x_{n}\right\rangle \geq 0, \quad \forall y \in C
$$

From (A2), we also have

$$
\varphi_{1}(y)-\varphi_{1}\left(t_{n}\right)+\left\langle A x_{n}, y-t_{n}\right\rangle+\frac{1}{r_{n}}\left\langle y-t_{n}, t_{n}-x_{n}\right\rangle \geq F_{1}\left(y, t_{n}\right), \quad \forall y \in C
$$

For $t$ with $0<t \leq 1$ and $y \in C$, let $y_{t}=t y+(1-t) z$. Since $y \in C$ and $z \in C$, we have $y_{t} \in C$. Then, we have

$$
\begin{aligned}
\left\langle y_{t}-t_{n_{i}}, A y_{t}\right\rangle \geq & \left\langle y_{t}-t_{n_{i}}, A y_{t}\right\rangle-\varphi_{1}\left(y_{t}\right)+\varphi_{1}\left(t_{n_{i}}\right)-\left\langle y_{t}-t_{n_{i}}, A x_{n_{i}}\right\rangle \\
& -\left\langle y_{t}-t_{n_{i}}, \frac{t_{n_{i}}-x_{n_{i}}}{r_{n_{i}}}\right\rangle+F_{1}\left(y_{t}, t_{n_{i}}\right) \\
= & \left\langle y_{t}-t_{n_{i}}, A y_{t}-A t_{n_{i}}\right\rangle+\left\langle y_{t}-t_{n_{i}}, A t_{n_{i}}-A x_{n_{i}}\right\rangle-\varphi_{1}\left(y_{t}\right)+\varphi_{1}\left(t_{n_{i}}\right) \\
& -\left\langle y_{t}-t_{n_{i}}, \frac{t_{n_{i}}-x_{n_{i}}}{r_{n_{i}}}\right\rangle+F_{1}\left(y_{t}, t_{n_{i}}\right) .
\end{aligned}
$$


Since $\left\|t_{n_{i}}-x_{n_{i}}\right\| \rightarrow 0$, we have $\left\|A t_{n_{i}}-A x_{n_{i}}\right\| \rightarrow 0$. Further, from an inverse-strongly monotonicity of $A$, we have $\left\langle y_{t}-t_{n_{i}}, A y_{t}-A t_{n_{i}}\right\rangle \geq 0$. So, from (A4) and the weak lower semicontinuity of $\varphi_{1},\left(t_{n_{i}}-x_{n_{i}}\right) / r_{n_{i}} \rightarrow 0$ and $t_{n_{i}} \rightarrow z$, we have at the limit

$$
\left\langle y_{t}-z, A y_{t}\right\rangle \geq-\varphi_{1}\left(y_{t}\right)+\varphi_{1}(z)+F_{1}\left(y_{t}, z\right)
$$

as $i \rightarrow \infty$. From (A1), (A4), and (3.54), we also get

$$
\begin{aligned}
0 & =F_{1}\left(y_{t}, y_{t}\right)+\varphi_{1}\left(y_{t}\right)-\varphi_{1}\left(y_{t}\right) \\
& \leq t F_{1}\left(y_{t}, y\right)+(1-t) F_{1}\left(y_{t}, z\right)+t \varphi_{1}(y)-(1-t) \varphi_{1}(z)-\varphi\left(y_{t}\right) \\
& =t\left[F_{1}\left(y_{t}, y\right)+\varphi_{1}(y)-\varphi_{1}\left(y_{t}\right)\right]+(1-t)\left[F_{1}\left(y_{t}, z\right)+\varphi_{1}(z)-\varphi_{1}\left(y_{t}\right)\right] \\
& \leq t\left[F_{1}\left(y_{t}, y\right)+\varphi_{1}(y)-\varphi_{1}\left(y_{t}\right)\right]+(1-t)\left\langle y_{t}-z, A y_{t}\right\rangle \\
& =t\left[F_{1}\left(y_{t}, y\right)+\varphi_{1}(y)-\varphi_{1}\left(y_{t}\right)\right]+(1-t) t\left\langle y-z, A y_{t}\right\rangle, \\
0 & \leq F_{1}\left(y_{t}, y\right)+\varphi_{1}(y)-\varphi_{1}\left(y_{t}\right)+(1-t)\left\langle y-z, A y_{t}\right\rangle .
\end{aligned}
$$

Letting $t \rightarrow 0$, we have, for each $y \in C$,

$$
F_{1}(z, y)+\varphi_{1}(y)-\varphi_{1}(z)+\langle y-z, A z\rangle \geq 0
$$

This implies that $z \in \operatorname{GMEP}\left(F_{1}, \varphi_{1}, A\right)$. By the same arguments, we can show that $z \in$ $\operatorname{GMEP}\left(F_{2}, \varphi_{2}, B\right)$.

Lastly, by the same proof of [39, Theorem 3.1, pages 346-347], we can show that $z \in$ $\mathrm{VI}(C, D)$ and $z \in \mathrm{VI}(C, E)$. Therefore, $z \in\left(\bigcap_{i=1}^{\infty} F\left(T_{i}\right)\right) \cap \operatorname{GMEP}\left(F_{1}, \varphi_{1}, A\right) \cap \operatorname{GMEP}\left(F_{2}, \varphi_{2}, B\right) \cap$ $\mathrm{VI}(C, D) \cap \mathrm{VI}(C, E)$; that is, $z \in \Theta$.

Noting that since $x_{n}=P_{C_{n}} x_{0}$, by $(2.4)$, we have

$$
\left\langle x_{0}-x_{n}, y-x_{n}\right\rangle \leq 0, \quad \forall y \in C_{n}
$$

Since $\Theta \subset C_{n}$ and by the continuity of inner product, we obtain from the above inequality that

$$
\left\langle x_{0}-z, y-z\right\rangle \leq 0, \quad \forall y \in C
$$

By (2.4), again, we conclude that $z=P_{\Theta} x_{0}$. This completes the proof.

Using Theorem 3.1, we obtain the following corollaries.

Corollary 3.2. Let $C$ be a nonempty closed convex subset of a real Hilbert Space $H$. Let $F_{1}, F_{2}$ be a bifunction of $C \times C$ into real numbers $\mathbb{R}$ satisfying (A1)-(A4), and let $\varphi_{1}, \varphi_{2}: C \rightarrow \mathbb{R} \cup\{+\infty\}$ be a proper lower semicontinuous and convex function. Let $A, B, D$, and $E$ be $\alpha, \beta, \delta$, and $\eta$-inversestrongly monotone mapping of $C$ into $H$, respectively. Let $T$ be nonexpansive mapping such that 
$\Theta:=F(T) \cap \operatorname{GMEP}\left(F_{1}, \varphi_{1}, A\right) \cap \operatorname{GMEP}\left(F_{2}, \varphi_{2}, B\right) \cap \operatorname{VI}(C, D) \cap \operatorname{VI}(C, E) \neq \emptyset$. Assume that either (B1) or (B2) holds. Let $\left\{x_{n}\right\}$ be a sequence generated by $x_{0} \in C, x_{1}=P_{C_{1}} x_{0}$ and

$$
\begin{gathered}
t_{n}=T_{\left.r_{n}, \varphi_{1}\right)}^{\left(F_{1}\right)}\left(x_{n}-r_{n} A x_{n}\right), \\
u_{n}=T_{S_{n}}^{\left(F_{2}, \varphi_{2}\right)}\left(x_{n}-s_{n} B x_{n}\right), \\
w_{n}=\xi_{n} P_{C}\left(u_{n}-\lambda_{n} D u_{n}\right)+\left(1-\xi_{n}\right) P_{C}\left(t_{n}-\mu_{n} E t_{n}\right), \\
y_{n}=\alpha_{n} x_{0}+\left(1-\alpha_{n}\right) T w_{n}, \\
C_{n+1}=\left\{z \in C_{n}:\left\|y_{n}-z\right\|^{2} \leq\left\|x_{n}-z\right\|^{2}+\alpha_{n}\left(\left\|x_{0}\right\|^{2}+2\left\langle w_{n}-x_{0}, z\right\rangle\right)\right\}, \\
x_{n+1}=P_{C_{n+1}} x_{0},
\end{gathered}
$$

for every $n \geq 0$, where $\left\{r_{n}\right\},\left\{s_{n}\right\} \subset(0, \infty), \lambda_{n} \in(0,2 \delta)$, and $\mu_{n} \in(0,2 \eta)$ satisfy the following conditions:

(i) $0<a \leq r_{n} \leq b<2 \alpha$,

(ii) $0<c \leq s_{n} \leq d<2 \beta$,

(iii) $\lim _{n \rightarrow \infty} \alpha_{n}=0$,

(iv) $\lim _{n \rightarrow \infty} \xi_{n}=\xi \in(0,1)$,

(v) $0<e \leq \lambda_{n} \leq f<2 \delta$,

(vi) $0<g \leq \mu_{n} \leq j<2 \eta$.

Then, $\left\{x_{n}\right\}$ converges strongly to $P_{\Theta} x_{0}$.

Proof. Taking $T_{i}=T$ for $i=1,2, \ldots$, to be nonexpansive mappings, in Theorem 3.1, we can conclude the desired conclusion easily. This completes the proof.

A mapping $T: C \rightarrow C$ is said to be a $\kappa$-strict pseudocontraction [40] if there exists a constant $0 \leq \kappa<1$ such that

$$
\|T x-T y\|^{2} \leq\|x-y\|^{2}+\kappa\|(I-T) x-(I-T) y\|^{2}, \quad \forall x, y \in C,
$$

where $I$ denotes the identity operator on $C$.

Lemma 3.3 (see [41]). Let $C$ be a nonempty closed convex subset of a real Hilbert space $H$, and let $T: C \rightarrow C$ be a $\kappa$-strict pseudocontraction. Define $S x: C \rightarrow C$ by $S x=\alpha x+(1-\alpha)$ Tx for each $x \in C$. Then, as $\alpha \in[\kappa, 1) S$ is nonexpansive such that $F(S)=F(T)$.

Using Theorem 3.1, we obtain the following result.

Theorem 3.4. Let $C$ be a nonempty closed convex subset of a real Hilbert Space $H$. Let $F_{1}, F_{2}$ be a bifunction of $C \times C$ into real numbers $\mathbb{R}$ satisfying (A1)-(A4), and let $\varphi_{1}, \varphi_{2}: C \rightarrow \mathbb{R} \cup\{+\infty\}$ be a proper lower semicontinuous and convex function. Let $A, B, D$, and $E$ be $\alpha, \beta, \delta$, and $\eta$-inversestrongly monotone mapping of $C$ into $H$, respectively. Let $T_{1}, T_{2}, \ldots, T_{N}$ be a finite family of $\kappa_{i^{-}}$ psuedocontractions such that $\Theta:=\bigcap_{i=1}^{N} F\left(T_{i}\right) \cap \operatorname{GMEP}\left(F_{1}, \varphi_{1}, A\right) \cap \operatorname{GMEP}\left(F_{2}, \varphi_{2}, B\right) \cap \operatorname{VI}(C, D) \cap$ $\operatorname{VI}(C, E) \neq \emptyset$. Define a mapping $T_{\mathcal{\kappa}_{i}}$ by $T_{\mathcal{\kappa}_{i}}=\kappa_{i} x+\left(1-\kappa_{i}\right) T_{i} x$ for all $x \in C, i \in\{1,2, \ldots, N\}$. Let 
$S_{n}$ be the S-mappings generated by $T_{\mathcal{K}_{1}}, T_{\mathcal{K}_{2}}, \ldots, T_{\mathcal{K}_{N}}$ and $\alpha_{n, i^{\prime}}^{1}, \alpha_{n, i^{\prime}}^{2}, \ldots, \alpha_{n, i^{i}}^{N}$. Assume that either (B1) or (B2) holds. Let $\left\{x_{n}\right\}$ be a sequence generated by $x_{0} \in C, C_{1, i}=C, C_{1}=\bigcap_{i=1}^{\infty} C_{1, i}, x_{1}=P_{C_{1}} x_{0}$ and

$$
\begin{gathered}
t_{n}=T_{\left.r_{n}, F_{1}\right)}^{\left(F_{1}\right)}\left(x_{n}-r_{n} A x_{n}\right), \\
u_{n}=T_{s_{n}}^{\left(F_{2}, \varphi_{2}\right)}\left(x_{n}-s_{n} B x_{n}\right), \\
w_{n}=\xi_{n} P_{C}\left(u_{n}-\lambda_{n} D u_{n}\right)+\left(1-\xi_{n}\right) P_{C}\left(t_{n}-\mu_{n} E t_{n}\right), \\
y_{n, i}=\alpha_{n, i} x_{0}+\left(1-\alpha_{n, i}\right) T_{i} w_{n}, \\
C_{n+1, i}=\left\{z \in C_{n, i}:\left\|y_{n, i}-z\right\|^{2} \leq\left\|x_{n}-z\right\|^{2}+\alpha_{n, i}\left(\left\|x_{0}\right\|^{2}+2\left\langle w_{n}-x_{0}, z\right\rangle\right)\right\}, \\
C_{n+1}=\bigcap_{i=1}^{\infty} C_{n+1, i}, \\
x_{n+1}=P_{C_{n+1}} x_{0},
\end{gathered}
$$

for every $n \geq 0$, where $\left\{r_{n}\right\},\left\{s_{n}\right\} \subset(0, \infty), \lambda_{n} \in(0,2 \delta)$, and $\mu_{n} \in(0,2 \eta)$ satisfy the following conditions:

(i) $0<a \leq r_{n} \leq b<2 \alpha$,

(ii) $0<c \leq s_{n} \leq d<2 \beta$,

(iii) $\lim _{n \rightarrow \infty} \alpha_{n, i}=0$,

(iv) $\lim _{n \rightarrow \infty} \xi_{n}=\xi \in(0,1)$,

(v) $0<e \leq \lambda_{n} \leq f<2 \delta$,

(vi) $0<g \leq \mu_{n} \leq j<2 \eta$.

Then, $\left\{x_{n}\right\}$ converges strongly to $P_{\Theta} x_{0}$.

Proof. From Theorem 3.1, $\left\{T_{i}\right\}_{i=1}^{N}$ is a finite family of $\kappa_{i}$-strict pseudocontraction. By Lemma 3.3, we have that $T_{\kappa_{i}}$ is nonexpansive mappings. The conclusion of Theorem 3.4 can be obtained from Theorem 3.1 immediately.

\section{Some Applications}

\subsection{Complementarity Problem}

Let $C$ be a nonempty closed and convex cone in $H$, and let $E$ be an operator of $C$ into $H$. We define the polar of $C$ in $H$ to be the set

$$
K^{*}:=\left\{y^{*} \in H:\left\langle x, y^{*}\right\rangle \geq 0, \forall x \in C\right\}
$$

Then, the element $u \in C$ is called a solution of the complementarity problem if

$$
E u \in K^{*}, \quad\langle u, E u\rangle=0 .
$$


The set of solution of the complementarity problem is denoted by $C^{\prime}(C, D), C^{\prime}(C, E)$. We will assume that $D, E$ satisfies the following conditions:

(E1) $D, E$ is a $\delta, \eta$-inverse-strongly monotone mapping, respectively,

(E2) $C^{\prime}(C, D), C^{\prime}(C, E) \neq \emptyset$.

(B1) For each $x \in H$ and $r>0$, there exist a bounded subset $D_{x} \subseteq C$ and $y_{x} \in C \cap \operatorname{dom}(\varphi)$ such that for any $z \in C \backslash D_{x}$,

$$
F\left(z, y_{x}\right)+\varphi\left(y_{x}\right)+\frac{1}{r}\left\langle y_{x}-z, z-x\right\rangle<\varphi(z)
$$

(B2) $C$ is a bounded set.

Corollary 4.1. Let $C$ be a nonempty closed convex subset of a real Hilbert Space $H$. Let $F_{1}, F_{2}$ be a bifunction of $C \times C$ into real numbers $\mathbb{R}$ satisfying (A1)-(A4), and let $\varphi_{1}, \varphi_{2}: C \rightarrow \mathbb{R} \cup\{+\infty\}$ be a proper lower semicontinuous and convex function. Let $A, B, D$, and $E$ be $\alpha, \beta, \delta$, and $\eta$-inversestrongly monotone mapping of $C$ into $H$, respectively. Let $T_{1}, T_{2}, \ldots$ be infinite nonexpansive mapping such that $\Theta:=\bigcap_{i=1}^{\infty} F\left(T_{i}\right) \cap \operatorname{GMEP}\left(F_{1}, \varphi_{1}, A\right) \cap \operatorname{GMEP}\left(F_{2}, \varphi_{2}, B\right) \cap C^{\prime}(C, D) \cap C^{\prime}(C, E) \neq \emptyset$. Assume that either (B1) or (B2) holds. Let $\left\{x_{n}\right\}$ be a sequence generated by $x_{0} \in C, C_{1, i}=C, C_{1}=$ $\bigcap_{i=1}^{\infty} C_{1, i}, x_{1}=P_{C_{1}} x_{0}$ and

$$
\begin{aligned}
& t_{n}=T_{r_{n}}^{\left(F_{1}, \varphi_{1}\right)}\left(x_{n}-r_{n} A x_{n}\right), \\
& u_{n}=T_{s_{n}}^{\left(F_{2}, \varphi_{2}\right)}\left(x_{n}-s_{n} B x_{n}\right), \\
& w_{n}=\xi_{n} P_{C}\left(u_{n}-\lambda_{n} D u_{n}\right)+\left(1-\xi_{n}\right) P_{C}\left(t_{n}-\mu_{n} E t_{n}\right), \\
& y_{n, i}=\alpha_{n, i} x_{0}+\left(1-\alpha_{n, i}\right) T_{i} w_{n} \\
& C_{n+1, i}=\left\{z \in C_{n, i}:\left\|y_{n, i}-z\right\|^{2} \leq\left\|x_{n}-z\right\|^{2}+\alpha_{n, i}\left(\left\|x_{0}\right\|^{2}+2\left\langle w_{n}-x_{0}, z\right\rangle\right)\right\}, \\
& C_{n+1}=\bigcap_{i=1}^{\infty} C_{n+1, i} \\
& x_{n+1}=P_{C_{n+1}} x_{0},
\end{aligned}
$$

for every $n \geq 0$, where $\left\{r_{n}\right\},\left\{s_{n}\right\} \subset(0, \infty), \lambda_{n} \in(0,2 \delta)$, and $\mu_{n} \in(0,2 \eta)$ satisfy the following conditions:

(i) $0<a \leq r_{n} \leq b<2 \alpha$,

(ii) $0<c \leq s_{n} \leq d<2 \beta$,

(iii) $\lim _{n \rightarrow \infty} \alpha_{n, i}=0$,

(iv) $\lim _{n \rightarrow \infty} \xi_{n}=\xi \in(0,1)$,

(v) $0<e \leq \lambda_{n} \leq f<2 \delta$,

(vi) $0<g \leq \mu_{n} \leq j<2 \eta$.

Then, $\left\{x_{n}\right\}$ converges strongly to $P_{\Theta} x_{0}$. 
Fixed Point Theory and Applications

Proof. Using Lemma 7.1.1 of [42], we have that $\operatorname{VI}(C, D)=C^{\prime}(C, D)$ and $\operatorname{VI}(C, E)=C^{\prime}(C, E)$. Hence, by Corollary 4.1, we can conclude the desired conclusion easily. This completes the proof.

\subsection{Optimization Problem}

In this section, we study a kind of multiobjective optimization problem by using the result of this paper. We will give an iterative algorithm of solution for the following optimization problem with nonempty set of solutions:

$$
\begin{array}{r}
\min h_{1}(x), \\
\min h_{2}(x), \\
x \in C,
\end{array}
$$

where $h(x)$ is a convex and lower semicontinuous functional, and define $C$ as a closed convex subset of a real Hilbert space $H$. We denote the set of solutions of (4.5) by $M\left(h_{1}\right)$ and $M\left(h_{2}\right)$. Let $F_{i}: C \times C \rightarrow \mathbb{R}$ be a bifunction defined by $F_{i}(x, y)=h_{i}(y)-h_{i}(x)$. We consider the equilibrium problem, it is obvious that $\operatorname{EP}\left(F_{i}\right)=M\left(h_{i}\right), i=1,2$. Therefore, from Theorem 3.1, we obtained the following corollary.

Corollary 4.2. Let $C$ be a nonempty closed convex subset of a real Hilbert Space $H$. Let $F_{1}, F_{2}$ be a bifunction of $C \times C$ into real numbers $\mathbb{R}$ satisfying (A1)-(A4), and let $\varphi_{1}, \varphi_{2}: C \rightarrow \mathbb{R} \cup\{+\infty\}$ be a proper lower semicontinuous and convex function. Let $A, B, D$, and $E$ be $\alpha, \beta, \delta$, and $\eta$-inversestrongly monotone mapping of $C$ into $H$, respectively. Let $T_{1}, T_{2}, \ldots$ be an infinite nonexpansive mapping such that $\Theta:=\bigcap_{i=1}^{\infty} F\left(T_{i}\right) \cap \operatorname{MEP}\left(F_{1}, \varphi_{1}\right) \cap \operatorname{MEP}\left(F_{2}, \varphi_{2}\right) \cap \operatorname{VI}(C, D) \cap \operatorname{VI}(C, E) \neq \emptyset$. Assume that either (B1) or (B2) holds. Let $\left\{x_{n}\right\}$ be a sequence generated by $x_{0} \in C, C_{1, i}=C, C_{1}=\bigcap_{i=1}^{\infty} C_{1, i}$, $x_{1}=P_{C_{1}} x_{0}$, and

$$
\begin{gathered}
h_{1}(t)-h_{1}\left(t_{n}\right)+\frac{1}{r_{n}}\left\langle t-t_{n}, t_{n}-x_{n}\right\rangle \geq 0, \quad \forall t \in C, \\
h_{2}(u)-h_{2}\left(u_{n}\right)+\frac{1}{s_{n}}\left\langle u-u_{n}, u_{n}-t_{n}\right\rangle \geq 0, \quad \forall u \in C, \\
w_{n}=\xi_{n} P_{C}\left(u_{n}-\lambda_{n} D u_{n}\right)+\left(1-\xi_{n}\right) P_{C}\left(t_{n}-\mu_{n} E t_{n}\right), \\
y_{n, i}=\alpha_{n, i} x_{0}+\left(1-\alpha_{n, i}\right) T_{i} w_{n}, \\
C_{n+1, i}=\left\{z \in C_{n, i}:\left\|y_{n, i}-z\right\|^{2} \leq\left\|x_{n}-z\right\|^{2}+\alpha_{n, i}\left(\left\|x_{0}\right\|^{2}+2\left\langle w_{n}-x_{0}, z\right\rangle\right)\right\}, \\
C_{n+1}=\bigcap_{i=1}^{\infty} C_{n+1, i} \\
x_{n+1}=P_{C_{n+1}} x_{0},
\end{gathered}
$$


for every $n \geq 0$, where $\left\{r_{n}\right\},\left\{s_{n}\right\} \subset(0, \infty), \lambda_{n} \in(0,2 \delta)$, and $\mu_{n} \in(0,2 \eta)$ satisfy the following conditions:

(i) $\lim _{n \rightarrow \infty} \alpha_{n, i}=0$,

(ii) $\lim _{n \rightarrow \infty} \xi_{n}=\xi \in(0,1)$,

(iii) $0<e \leq \lambda_{n} \leq f<2 \delta$,

(iv) $0<g \leq \mu_{n} \leq j<2 \eta$.

Then, $\left\{x_{n}\right\}$ converges strongly to $P_{\Theta} x_{0}$.

Proof. From Theorem 3.1, put $F_{1}\left(t_{n}, t\right)=h_{1}(t)-h_{1}\left(t_{n}\right), F_{2}\left(u_{n}, u\right)=h_{2}(u)-h_{2}\left(u_{n}\right)$, and $A, B, \varphi_{1}, \varphi_{2} \equiv 0$. The conclusion of Corollary 4.2 can be obtained from Theorem 3.1 immediately.

\subsection{Minimization Problem}

In this section, we study the problem for finding a minimizer of a continuously Frèchet differentiable convex functional in a Hilbert space.

First, we use the following lemma in our result.

Lemma 4.3 (see [43]). Let $E$ be a Banach space, let $f$ be a continuously Frèchet differentiable convex functional on $E$, and let $\nabla f$ be the gradient of $f$. If $\nabla f$ is $(1 / \alpha)$-Lipschitz continuous, then $\nabla f$ is an $\alpha$-inverse-strongly monotone.

Let $f_{1}, f_{2}$ be functionals on $H$ which satisfies the following conditions:

(C1) let, $f_{1}, f_{2}$ be a continuously Frèchet differentiable convex functional on $H$, and let, $\nabla f_{1}, \nabla f_{2}$ be $(1 / \delta),(1 / \eta)$-Lipschitz continuous, respectively,

(C2) $\left(\nabla f_{1}\right)^{-1} 0=\left\{z_{1} \in H: f_{1}\left(z_{1}\right)=\min _{y_{1} \in H} f_{1}\left(y_{1}\right)\right\} \neq \emptyset$ and $\left(\nabla f_{2}\right)^{-1} 0=\left\{z_{2} \in H: f_{2}\left(z_{2}\right)=\right.$ $\left.\min _{y_{2} \in H} f_{2}\left(y_{2}\right)\right\} \neq \emptyset$.

Corollary 4.4. Let $H$ be a real Hilbert Space. Let $F_{1}, F_{2}$ be a bifunction of $H \times H$ into real numbers $\mathbb{R}$ satisfying (A1)-(A4), and let $\varphi_{1}, \varphi_{2}: C \rightarrow \mathbb{R} \cup\{+\infty\}$ be a proper lower semicontinuous and convex function. Let $A, B$ be $\alpha, \beta$-inverse-strongly monotone mapping of $H$ into $H$, respectively. Let $T_{1}, T_{2}, \ldots$ be infinite nonexpansive mappings. Let $f_{1}, f_{2}$ be functionals on $H$ which satisfies the conditions 
(C1) and (C2). Suppose that $\Theta:=\bigcap_{i=1}^{\infty} F\left(T_{i}\right) \cap \operatorname{GMEP}\left(F_{1}, \varphi_{1}\right) \cap \operatorname{GMEP}\left(F_{2}, \varphi_{2}\right) \cap\left(\nabla f_{1}\right)^{-1} 0 \cap$ $\left(\nabla f_{2}\right)^{-1} 0 \neq \emptyset$. Assume that either (B1) or (B2) holds. Let $\left\{x_{n}\right\}$ be a sequence generated by $x_{0} \in C$, $C_{1, i}=C, C_{1}=\bigcap_{i=1}^{\infty} C_{1, i}, x_{1}=P_{C_{1}} x_{0}$, and

$$
\begin{gathered}
t_{n}=T_{r_{n}}^{\left(F_{1}, \varphi_{1}\right)}\left(x_{n}-r_{n} A x_{n}\right), \\
u_{n}=T_{s_{n}}^{\left(F_{2}, \varphi_{2}\right)}\left(x_{n}-s_{n} B x_{n}\right), \\
w_{n}=\xi_{n}\left(u_{n}-\lambda_{n} \nabla f_{1}\left(u_{n}\right)\right)+\left(1-\xi_{n}\right)\left(t_{n}-\mu_{n} \nabla f_{2}\left(t_{n}\right)\right), \\
y_{n, i}=\alpha_{n, i} x_{0}+\left(1-\alpha_{n, i}\right) T_{i} w_{n}, \\
C_{n+1, i}=\left\{z \in C_{n, i}:\left\|y_{n, i}-z\right\|^{2} \leq\left\|x_{n}-z\right\|^{2}+\alpha_{n, i}\left(\left\|x_{0}\right\|^{2}+2\left\langle w_{n}-x_{0}, z\right\rangle\right)\right\}, \\
C_{n+1}=\bigcap_{i=1}^{\infty} C_{n+1, i}, \\
x_{n+1}=P_{C_{n+1}} x_{0},
\end{gathered}
$$

for every $n \geq 0$, where $\left\{r_{n}\right\},\left\{s_{n}\right\} \subset(0, \infty), \lambda_{n} \in(0,2 \delta)$, and $\mu_{n} \in(0,2 \eta)$ satisfying the following conditions:
(i) $0<a \leq r_{n} \leq b<2 \alpha$,
(ii) $0<c \leq s_{n} \leq d<2 \beta$,
(iii) $\lim _{n \rightarrow \infty} \alpha_{n, i}=0$,
(iv) $\lim _{n \rightarrow \infty} \xi_{n}=\xi \in(0,1)$,
(v) $0<e \leq \lambda_{n} \leq f<2 \delta$,
(vi) $0<g \leq \mu_{n} \leq j<2 \eta$.

Then, $\left\{x_{n}\right\}$ converges strongly to $P_{\Theta} x_{0}$.

Proof. We know form condition (C1) and Lemma 4.3 that $\nabla f_{1}, \nabla f_{2}$ is a $\delta, \eta$-inverse-strongly monotone operator from $H$ in to itself, respectively. The conclusion of Corollary 4.4 can be obtained from Theorem 3.1 immediately.

\section{Acknowledgments}

The authors are grateful to the anonymous referees for their helpful comments which improved the presentation of the original version of this work. The authors would like to thank The National Research University Project of Thailand's Office of the Higher Education Commission for financial support (under the NRU-CSEC Project no. 54000267). Furthermore, P. Kumam's research supported by the Commission on Higher Education and the Thailand Research Fund under Grant no. MRG5380044.

\section{References}

[1] W. Takahashi, Nonlinear Functional Analysis, Yokohama Publishers, Yokohama, Japan, 2000. 
[2] E. Blum and W. Oettli, "From optimization and variational inequalities to equilibrium problems," The Mathematics Student, vol. 63, no. 1-4, pp. 123-145, 1994.

[3] O. Chadli, S. Schaible, and J. C. Yao, "Regularized equilibrium problems with application to noncoercive hemivariational inequalities," Journal of Optimization Theory and Applications, vol. 121, no. 3, pp. 571-596, 2004.

[4] O. Chadli, N. C. Wong, and J. C. Yao, "Equilibrium problems with applications to eigenvalue problems," Journal of Optimization Theory and Applications, vol. 117, no. 2, pp. 245-266, 2003.

[5] I. V. Konnov, S. Schaible, and J. C. Yao, "Combined relaxation method for mixed equilibrium problems," Journal of Optimization Theory and Applications, vol. 126, no. 2, pp. 309-322, 2005.

[6] A. Moudafi and M. Théra, "Proximal and dynamical approaches to equilibrium problems," in IllPosed Variational Problems and Regularization Techniques, vol. 477 of Lecture Notes in Economics and Mathematical Systems, pp. 187-201, Springer, Berlin, Germany, 1999.

[7] L.-C. Zeng, S.-Y. Wu, and J.-C. Yao, "Generalized KKM theorem with applications to generalized minimax inequalities and generalized equilibrium problems," Taiwanese Journal of Mathematics, vol. 10, no. 6, pp. 1497-1514, 2006.

[8] L.-C. Ceng and J.-C. Yao, "A hybrid iterative scheme for mixed equilibrium problems and fixed point problems," Journal of Computational and Applied Mathematics, vol. 214, no. 1, pp. 186-201, 2008.

[9] W. Takahashi and M. Toyoda, "Weak convergence theorems for nonexpansive mappings and monotone mappings," Journal of Optimization Theory and Applications, vol. 118, no. 2, pp. 417-428, 2003.

[10] S. Takahashi and W. Takahashi, "Viscosity approximation methods for equilibrium problems and fixed point problems in Hilbert spaces," Journal of Mathematical Analysis and Applications, vol. 331, no. 1, pp. 506-515, 2007.

[11] R. S. Burachik, J. O. Lopes, and G. J. P. Da Silva, “An inexact interior point proximal method for the variational inequality problem," Computational \& Applied Mathematics, vol. 28, no. 1, pp. 15-36, 2009.

[12] Y. J. Cho, N. Petrot, and S. Suantai, "Fixed point theorems for nonexpansive mappings with applications to generalized equilibrium and system of nonlinear variational inequalities problems," Journal of Nonlinear Analysis and Optimization, vol. 1, no. 1, pp. 45-53, 2010.

[13] S. D. Flåm and A. S. Antipin, "Equilibrium programming using proximal-like algorithms," Mathematical Programming, vol. 78, no. 1, pp. 29-41, 1997.

[14] T. Jitpeera and P. Kumam, "A composite iterative method for generalized mixed equilibrium problems and variational inequality problems," Journal of Computational Analysis and Applications, vol. 13, no. 2, pp. 345-361, 2011.

[15] T. Jitpeera and P. Kumam, "An extragradient type method for a system of equilibrium problems, variational inequality problems and fixed points of finitely many nonexpansive mappings," Journal of Nonlinear Analysis and Optimization, vol. 1, pp. 71-91, 2010.

[16] C. Jaiboon and P. Kumam, "Strong convergence for generalized equilibrium problems, fixed point problems and relaxed cocoercive variational inequalities," Journal of Inequalities and Applications, vol. 2010, Article ID 728028, 43 pages, 2010.

[17] C. Jaiboon and P. Kumam, "A general iterative method for addressing mixed equilibrium problems and optimization problems," Nonlinear Analysis. Theory, Methods E Applications, vol. 73, no. 5, pp. 1180-1202, 2010.

[18] P. Kumam, "A hybrid approximation method for equilibrium and fixed point problems for a monotone mapping and a nonexpansive mapping," Nonlinear Analysis. Hybrid Systems, vol. 2, no. 4, pp. 1245-1255, 2008.

[19] P. Kumam, "Strong convergence theorems by an extragradient method for solving variational inequalities and equilibrium problems in a Hilbert space," Turkish Journal of Mathematics, vol. 33, no. 1, pp. 85-98, 2009.

[20] P. Kumam, "A new hybrid iterative method for solution of equilibrium problems and fixed point problems for an inverse strongly monotone operator and a nonexpansive mapping," Journal of Applied Mathematics and Computing, vol. 29, no. 1-2, pp. 263-280, 2009.

[21] P. Kumam and C. Jaiboon, "A new hybrid iterative method for mixed equilibrium problems and variational inequality problem for relaxed cocoercive mappings with application to optimization problems," Nonlinear Analysis. Hybrid Systems, vol. 3, no. 4, pp. 510-530, 2009.

[22] P. Kumam and C. Jaiboon, "A system of generalized mixed equilibrium problems and fixed point problems for pseudocontractive mappings in Hilbert spaces," Fixed Point Theory and Applications, vol. 2010, Article ID 361512, 33 pages, 2010. 
[23] P. Kumam and P. Katchang, "A viscosity of extragradient approximation method for finding equilibrium problems, variational inequalities and fixed point problems for nonexpansive mappings," Nonlinear Analysis. Hybrid Systems, vol. 3, no. 4, pp. 475-486, 2009.

[24] W. Kumam, C. Jaiboon, P. Kumam, and A. Singta, "A shrinking projection method for generalized mixed equilibrium problems, variational inclusion problems and a finite family of quasi-nonexpansive mappings," Journal of Inequalities and Applications, vol. 2010, Article ID 458247, 25 pages, 2010.

[25] Z. Wang and Y. Su, "Strong convergence theorems of common elements for equilibrium problems and fixed point problems in Banach paces," Journal of Application Mathematics and Informatics, vol. 28, no. 3-4, pp. 783-796, 2010.

[26] R. Wangkeeree and R. Wangkeeree, "Strong convergence of the iterative scheme based on the extragradient method for mixed equilibrium problems and fixed point problems of an infinite family of nonexpansive mappings," Nonlinear Analysis. Hybrid Systems, vol. 3, no. 4, pp. 719-733, 2009.

[27] R. Wangkeeree, N. Petrot, P. Kumam, and C. Jaiboon, "Convergence theorem for mixed equilibrium and variational inequality problems for relaxed cocoercive mappings," Journal of Computational Analysis and Applications, vol. 13, no. 3, pp. 425-449, 2011.

[28] Y. Yao, M. A. Noor, S. Zainab, and Y.-C. Liou, "Mixed equilibrium problems and optimization problems," Journal of Mathematical Analysis and Applications, vol. 354, no. 1, pp. 319-329, 2009.

[29] Y. Yao, Y. J. Cho, and R. Chen, "An iterative algorithm for solving fixed point problems, variational inequality problems and mixed equilibrium problems," Nonlinear Analysis. Theory, Methods $\mathcal{E}$ Applications, vol. 71, no. 7-8, pp. 3363-3373, 2009.

[30] J.-C. Yao and O. Chadli, "Pseudomonotone complementarity problems and variational inequalities," in Handbook of Generalized Convexity and Generalized Monotonicity, vol. 76, pp. 501-558, Springer, New York, NY, USA, 2005.

[31] L. C. Zeng, S. Schaible, and J. C. Yao, "Iterative algorithm for generalized set-valued strongly nonlinear mixed variational-like inequalities," Journal of Optimization Theory and Applications, vol. 124, no. 3, pp. 725-738, 2005.

[32] W. Takahashi, Y. Takeuchi, and R. Kubota, "Strong convergence theorems by hybrid methods for families of nonexpansive mappings in Hilbert spaces," Journal of Mathematical Analysis and Applications, vol. 341, no. 1, pp. 276-286, 2008.

[33] A. Kangtunyakarn, "Iterative methods for finding common solution of generalized equilibrium problems and variational inequality problems and fixed point problems of a finite family of nonexpansive mappings," Fixed Point Theory and Applications, vol. 2010, Article ID 836714, 29 pages, 2010.

[34] Y. Shehu, "Strong convergence theorems for family of nonexpansive mappings and sys- tem of generalized mixed equilibrium problems and variational inequality problems," International Journal of Mathematics and Mathematical Sciences. In press.

[35] W. Chantarangsi, C. Jaiboon, and P. Kumam, "A viscosity hybrid steepest descent method for generalized mixed equilibrium problems and variational inequalities for relaxed cocoercive mapping in Hilbert spaces," Abstract and Applied Analysis, vol. 2010, Article ID 390972, 39 pages, 2010.

[36] P. L. Combettes and S. A. Hirstoaga, "Equilibrium programming in Hilbert spaces," Journal of Nonlinear and Convex Analysis, vol. 6, no. 1, pp. 117-136, 2005.

[37] J.-W. Peng, Y.-C. Liou, and J.-C. Yao, “An iterative algorithm combining viscosity method with parallel method for a generalized equilibrium problem and strict pseudocontractions," Fixed Point Theory and Applications, vol. 2009, Article ID 794178, 21 pages, 2009.

[38] H.-K. Xu, "Viscosity approximation methods for nonexpansive mappings," Journal of Mathematical Analysis and Applications, vol. 298, no. 1, pp. 279-291, 2004.

[39] H. liduka and W. Takahashi, "Strong convergence theorems for nonexpansive mappings and inversestrongly monotone mappings," Nonlinear Analysis. Theory, Methods E Applications, vol. 61, no. 3, pp. 341-350, 2005.

[40] F. E. Browder and W. V. Petryshyn, "Construction of fixed points of nonlinear mappings in Hilbert space," Journal of Mathematical Analysis and Applications, vol. 20, pp. 197-228, 1967.

[41] H. Zhou, "Convergence theorems of fixed points for k-strict pseudo-contractions in Hilbert spaces," Nonlinear Analysis. Theory, Methods E Applications, vol. 69, no. 2, pp. 456-462, 2008.

[42] W. Takahashi, "Weak and strong convergence theorems for families of nonexpansive mappings and their applications," Annales Universitatis Mariae Curie-Skłodowska, vol. 51, no. 2, pp. 277-292, 1997.

[43] J.-B. Baillon and G. Haddad, "Quelques propriétés des opérateurs angle-bornés et $n$-cycliquement monotones," Israel Journal of Mathematics, vol. 26, no. 2, pp. 137-150, 1977. 